
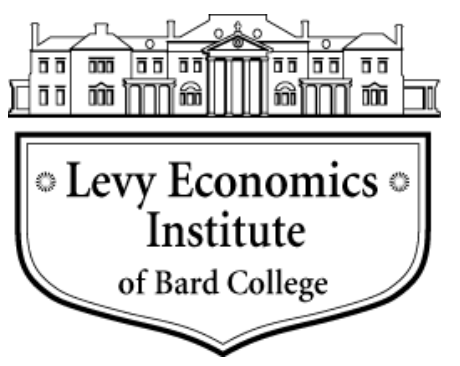

Working Paper No. 964

\title{
Budget Credibility of Subnational Governments: Analyzing the Fiscal Forecasting Errors of 28 States in India
}

\author{
by \\ Lekha Chakraborty \\ Levy Economics Institute of Bard College \\ and National Institute of Public Finance and Policy (NIPFP) \\ Pinaki Chakraborty \\ Levy Economics Institute of Bard College \\ and National Institute of Public Finance and Policy (NIPFP) \\ and \\ Ruzel Shrestha \\ National Institute of Public Finance and Policy (NIPFP)
}

June 2020

\begin{abstract}
* This consolidated paper was prepared as part of "Public Finance Innovations" project under the Gates Foundation. Special Thanks to Divy Rangan (NIPFP) for research support.

The Levy Economics Institute Working Paper Collection presents research in progress by Levy Institute scholars and conference participants. The purpose of the series is to disseminate ideas to and elicit comments from academics and professionals.
\end{abstract}

Levy Economics Institute of Bard College, founded in 1986, is a nonprofit, nonpartisan, independently funded research organization devoted to public service. Through scholarship and economic research it generates viable, effective public policy responses to important economic problems that profoundly affect the quality of life in the United States and abroad.

\author{
Levy Economics Institute \\ P.O. Box 5000 \\ Annandale-on-Hudson, NY 12504-5000 \\ http://www.levyinstitute.org
}

Copyright (C) Levy Economics Institute 2020 All rights reserved

ISSN 1547-366X 


\begin{abstract}
Budget credibility, or the ability of governments to accurately forecast macro-fiscal variables, is crucial for effective public finance management. Fiscal marksmanship analysis captures the extent of errors in the budgetary forecasting. The fiscal rules can determine fiscal marksmanship, as effective fiscal consolidation procedures affect the fiscal behavior of the states in conducting the budgetary forecasts. Against this backdrop, applying Theil's technique, we analyze the fiscal forecasting errors for 28 states (except Telangana) in India for the period 2011-16. There is a heterogeneity in the magnitude of errors across subnational governments in India. The forecast errors in revenue receipts have been greater than revenue expenditure. Within revenue receipts, the errors are more significantly pronounced in the grants component. Within expenditure budgets, the errors in capital spending are found to be greater than revenue spending in all the states. Partitioning the sources of errors, we identified that the errors were more broadly random than due to systematic bias, except for a few crucial macro-fiscal variables where improving the forecasting techniques can provide better estimates.
\end{abstract}

KEYWORDS: Forecast Errors; Fiscal Policies; Fiscal Forecasting; Political Economy; Fiscal Marksmanship

JEL CLASSIFICATIONS: H6; E62; C53 
Budget credibility, or a government's ability to accurately forecast macro-fiscal variables, is integral to public financial management. Fiscal marksmanship captures the extent of errors in the budgetary forecasting. Fiscal rules can determine fiscal marksmanship, as effective fiscal consolidation procedures affect the fiscal behavior of the states. Even logical and well-written fiscal rules require justification, given that constraining a government's ability to practice fiscal policy has obvious disadvantages as well (Auerbach 2017). Against this backdrop, we analyze the errors in the budget forecasts in India at the state level for the period 2011-16. The Fiscal Responsibility and Budget Management (FRBM) Act stipulated that states should maintain a threshold fiscal-deficit-to-GDP ratio of 3 percent except for in West Bengal, Kerala, and Punjab (Ministry of Finance 2017). FRBM compliance by the states has been rewarded with performance-incentive grants from the central government; therefore two issues are relevant to analyze here: (i) the credibility of budget forecasts and (ii) if there are any changes in a state's fiscal behavior ex post fiscal rules.

Technically, the revenue and expenditure forecasts are initially made in the finance minister's annual budget speech as "budget estimates"; these forecasts are revised after a year and published as "revised estimates." The finance accounts of the states, with a lag of one or two years, provides the actual figures for audited revenue and expenditure. There is a high likelihood of huge deviations between these three stages.

We examine these deviations in macro-fiscal variables for 28 states (except Telangana) in India by employing a technique that estimates the magnitude and sources of forecast errors. The paper is organized in six sections. Section 1 explains what forecast errors are and why studying them is important. Section 2 reviews the fiscal marksmanship analysis. Section 3 explains the data sources and measurement issues. Section 4 presents the magnitude of errors using simple statistical tools. Section 5 carries out the application of the Theil's U techniques for the evaluation of fiscal marksmanship and identifies the systemic and random components of forecast errors for all Indian states. Section 6 concludes and draws policy implications. 


\section{WHAT IS FORECAST ERROR AND WHY IS ANALYZING FORECAST ERROR IMPORTANT?}

Any budget has three sets of numbers: the budget estimates for the current year, the revised estimates for the ensuing year, and the actuals. The credibility of the budget depends on the quality of the budgetary estimates.

There can be various issues arising if the government estimates are inaccurate, which at times can have unintended/adverse macroeconomic consequences. In cases where the actual expenditure exceeds the budgeted, there would be an unanticipated need for financing the deficit. Conversely, if the actual expenditure is less than the budgeted, then there would be idle resources that could otherwise be put to productive use. Therefore, accurate forecasts are quintessential for proper budget implementation.

Accurate fiscal forecasts are also important for fiscal management. For instance, if a country wants to reduce its fiscal deficit, one needs to rely on the accuracy of the budgetary estimates of its revenues and expenditures. Generally, the budgetary estimates will consist of errors (i.e., the forecasts would deviate from the actual values). However, not all errors can be treated similarly. Primarily one can distinguish between systematic errors and random errors. The systematic errors can be improved upon by incorporating additional relevant variables or even factoring in the variations in the different variables involved. Conversely, random errors are the errors that cannot be improved upon using better forecasting methodologies/techniques and are because of unanticipated and exogenous shocks that are out of the forecaster's control. Therefore, credible budgetary forecasts would have a higher proportion of random errors compared to systematic errors. It is only when the estimates are credible that one can maintain a desired level of fiscal deficit.

Theil's index is used in the literature to assess the extent of errors. To know the composition of errors, we break down the error into systematic errors and random errors. If the systematic component of error is high, one can improve the forecasting by improving the forecasting method. This can be done by adding more variables into the forecasting model or also by incorporating the fluctuations in the variables in the model. In case the random error is high, one 
cannot improve the forecasting further and the model used to estimate the error is a good model (Theil 1958).

Effective fiscal consolidation at subnational levels of government requires a high degree of accuracy in forecasting tax revenue and in estimating public expenditure. Fiscal marksmanship is an exercise for examining the degree of correspondence between the actual and forecasted revenue and expenditure that will aid in assessing the extent of errors and also the composition of errors. Fiscal marksmanship is significant because the revenue projections/forecasting determine the extent of borrowing requirements to finance public expenditure. Public expenditure compression — the significant deviation between what is budgeted and what is actually spent—-to meet the FRBM targets also has adverse macroeconomic consequences.

\section{A REVIEW OF FISCAL MARKSMANSHIP ANALYSIS}

The political economy of budget deficits and other macro-fiscal variables started gaining attention in the 1990s (Alesina and Perotti 1995; Blanchard 1990). However, one of the earlier discussions of fiscal forecast errors was made by Allan (1965) in the case of Britain. According to Allan, fiscal marksmanship was important during that time was because the margin for error was limited given the tradeoff between inflation and full employment. In such a scenario, accurate predictions of budgetary estimates were important for meeting fiscal policy targets of full employment without undesirably high inflation. Davis (1980), following up on Allan's study, used a longer time series (from 1951 to 1978).

Auld (1970) has done a fiscal marksmanship exercise for Canada for the postwar period (through 1968). Auld says that if the government is to finance its long-range programs, accurate predictions are important. Morrison (1986) has done a fiscal marksmanship exercise in the United States for the years 1950-83. Cassidy, Kamlet, and Nagin (1989) analyzed the revenue forecast biases in the context of Europe. The expectations that macro-fiscal variables may be subject to error has been recognized as an important part of most explanations of the changes in the level of economic activity (Muth 1961). Good fiscal marksmanship can be one important piece of available information rational agents must consider in forming expectations. The 
significant variations between actual revenue and expenditure from the forecasted budgetary magnitudes could be an indicative of nonoptimization or nonattainment of set fiscal policy objectives. In this context, the role of budget estimates needs to be emphasized as what Davis (1980) refers to as fiscal signals, noting that budget estimates have an important "signal effect" for outside forecasters and analysts, with particular attention in recent years focused on the estimated borrowing requirement. If expectations are rational rather than adaptive, it is the estimate of taxes and public expenditure in any given budget — the ex ante data, not the observed data - that will be used by forward-looking private agents who base their decisions in whole or in part on fiscal variables (Morrison 1986).

In the context of the eurozone, Brück and Stephan (2005) have estimated the political economy determinants of budget deficit forecast errors. Their findings show that politics, electoral cycles, and the institutional design of governments affect the quality of fiscal forecasts. Their findings against the backdrop of the Stability and Growth Pact (SGP) ${ }^{1}$ suggest malign incentives for "unobservable fiscal effort" (Beetsma and Jensen 2004) by eurozone governments (compared to other OECD governments) in reporting their budget deficits prior to elections. They explained the fiscal behavior under three cycles - an electoral forecast cycle, a partisan forecast cycle, and an institutional cycle ${ }^{2}$ - applying panel econometric techniques to the analysis of forecast errors of both eurozone and non-eurozone OECD economies. Their findings suggest that the forecast errors align with election cycles in eurozone countries.

Rullán and Villalonga (2018), in the context of the SGP, have examined the relationship between fiscal rules and budgetary forecasts by analyzing the significance of political and institutional

\footnotetext{
${ }^{1}$ The SGP is a set of rules devised by the European Commission to ensure that countries in the European Union pursue sound public finances and coordinate their fiscal policies.

${ }^{2}$ They emphasized that in an electoral forecast cycle, the election date determines the nature of government spending and taxation plans; for instance, government may increase public expenditure and revise taxation plans prior to election date and manipulate the emerging budget deficit until after the elections. In a partisan forecast cycle, they have elaborated that a cyclical behavior derives from different preferences of the political parties and their respective voters. The quality of budget deficit forecasts in such a cycle depends on the political orientation of a government; for instance, the left-wing (right-wing) governments pursue employment (price stability) at the expense of price stability (employment), which means that tax revenues are more (less) difficult to forecast. In an institutional forecast cycle, they elaborated that the institutions of governance create incentives for manipulating budget deficit forecasts; for instance, the deficit forecasts of a coalition or minority government and a single-party majority government may not be the same. Artis and Marcellino (2001) also analyzed forecast errors in OECD countries.
} 
variables in the eurozone. Their findings show that the level of public sector debt is crucial in explaining budgetary forecast errors. The electoral mandate, political orientation of ruling parties, tax autonomy, and per capita revenue are the other significant determinants of forecast errors. This study took the literature forward to subnational tiers of government in 15 European countries, unlike the earlier studies in the context of eurozone that confined their analysis to a macroeconomic perspective at the national government levels. The SGP therefore creates incentives for creative budgetary deficit forecasts prior to election cycles (Strauch, Hallerberg, and Hagen 2004).

Giuriato, Cepparulo, and Barberi (2016) analyzed the quality of fiscal forecasts of 13 eurozone countries by using annual forecasts for the period 1999-2013 against the backdrop of the stability and convergence programmes. They found that if fiscal rules counter the executive's monopoly on fiscal forecasting, strengthening the legislature's formal powers negatively influences the fiscal forecast accuracy. Pina and Venes (2011) analyzed the budget balance forecasts prepared by 15 European countries in their Excessive Deficit Procedure (EDP) reporting. They found that growth surprises, fiscal institutions, elections cycles, forms of fiscal governance, and numerical expenditure rules (unlike deficit and debt rules) affect the forecast errors.

There have been a number of fiscal marksmanship exercises in the case of India (Bhattacharya and Kumari 1988). In one of the earlier attempts at analyzing budgetary estimates in India (for the period 1956-64), Samuel and Rangarajan (1974) undertook an analysis of two components of the state and union budgets' capital expenditure on construction and industrial development (the analysis was limited to these two because of the scope of the subject matter they were dealing with). In this study, the analysis of forecasting errors was based largely on graphs plotting the actual expenditure and the budget estimates. In their analysis, it is stated that while in both components the central government's budget estimate was more accurate compared to the state's, this difference was attributed to the difference in budgetary process's efficiency.

Asher (1978) performed a more comprehensive fiscal marksmanship exercise for India for the period 1967-76 for both the revised and budget estimates. The study showed that during that 
period, both the revenues and expenditures were consistently underestimated. However, it was observed that the extent of the error on the expenditure side was larger.

Chakrabarty and Varghese (1982) have used data from 1970-80. One of the major findings of that study was that both revenues and expenditure are underestimated. Pattnaik (1990) has done a fiscal marksmanship exercise using Theil's index for the period 1951-89. The study observes that the errors in the revised estimates are lower than the errors in the budget estimates (although there are large errors in both). It stated that the errors in the estimates are largely systematic in nature for both the entire time period as well as for smaller time periods within the whole (the systematic errors were greatest for the period 1981-89).

More recent studies on fiscal marksmanship in India have a different conclusion. A study done by Nitin and Roy (2015) using data from 1990-2012 observes that the source of error in components such as tax revenue, nontax revenue, interest payments, defense revenue expenditure, and fiscal deficit were primarily due to random error (defined in their paper as the proportion of the random error is greater than the bias components or the error in variance). The rest of the components - such as subsidy expenditures, capital expenditure, and nondebt capital receipts - had a higher systematic error (mean error and slope error). A very interesting point made in the paper is that while there is an attempt to have fiscal consolidation by controlling expenditure, the predictability of expenditure is quite low compared to revenue. In a similar study, Chakraborty and Sinha (2018) undertook a fiscal marksmanship exercise for the period 1990-2017 and have come up with a similar conclusion.

A trend that is observed based on the empirical literature from 1951 to 1990 is that the systematic component of the error was higher, while from 1990 to 2017 the random component is higher. It is worth noting that these above studies are based on the federal government's data. Shrestha and Chakraborty (2019) is the only study that has examined the fiscal marksmanship in the context of India's states. Their study focused on Kerala and identified forecast errors with respect to tax revenue projections.

In the recent empirical literature, the fiscal forecast errors are analyzed against the backdrop of fiscal rules. The political economy of fiscal forecasts at the subnational level depends on the tax 
autonomy and the nature of the intergovernmental fiscal transfer mechanism. The tax autonomy is heterogeneous across states. The intergovernmental fiscal transfers may be progressive if the transfer is designed to offset the interstate fiscal disabilities.

In India, the Finance Bill 2018 has incorporated a few clauses (clauses 207-10) to amend the FRBM Act of 2003, with special emphasis on the elimination of references to "revenue balance" (i.e., revenue receipts $=$ revenue expenditure) and using fiscal deficit as an operational parameter (Chakraborty and Chakraborty 2018). Against these policy changes, it is pertinent to analyze the impact of fiscal rules on fiscal marksmanship of macro-fiscal variables in India. Buiter and Patel (2011) have analyzed fiscal rules in India, however the effect of fiscal rules on fiscal marksmanship in the context of India has not been analyzed. As mentioned above, Nitin and Roy (2014) have analyzed the normative fiscal assessments of India's Finance Commission, and realization of fiscal policy with regard to the central government's finances over the period 1990-2012.

The recent empirical literature on fiscal marksmanship is highly confined to the Indian national government's forecast errors (Chakraborty and Sinha 2018; Nitin and Roy 2014). There has been virtually no effort to undertake a fiscal marksmanship exercise at the state level. In this paper, we attempt to do a fiscal marksmanship exercise at the state level for the period 2010-16, analyzing the magnitude of the states' errors and subsequently examining the nature of the errors. This is done in two ways: first we check whether the errors are overestimates or underestimates, and then we check the extent of systematic and random components in these fiscal forecast errors.

\section{DATA AND MEASUREMENT ISSUES}

The data is organized from the finance accounts of various states and the Central Statistics Office (CSO). The forecast error is defined as the deviation between what is predicted as budget estimates (BE) or revised estimates (RE) and what is actual. The summary statistics usually used to measure forecasting errors in the empirical literature are follow Cabanillas and Terzi (2012). 


\section{The Mean Error}

The mean error (ME) refers to the average difference between the forecast and the actual. The ME has been calculated by taking the average of the difference between the predicted values (of both $\mathrm{BE}$ and RE) and the actuals over the period 2011-16. We have divided the ME by the sum of the actuals for the reference period. The ME is a crude measure of the forecast's quality, as positive and negative errors can offset each other, thereby not giving us the exact magnitude of error. However, the ME is an indicator of possible bias in the forecast.

\section{The Root Mean Square Error}

The root mean squared error (RMSE) is a measure of the relative size of the forecast error. In this paper, to calculate the RMSE, the mean squared error (MSE) is taken over the reference period after which the square root of the MSE is calculated. While this will give us the magnitude of error, it will not give any information on the direction of the error, i.e., whether the error is positive or negative. We have taken the RMSE as a proportion of the sum of actuals of the reference period. It reflects the fact that large forecast errors are more significant than small differences.

\section{Theil's Inequality Coefficients (U)}

Theil's inequality coefficient (U) is used to analyze the measure of accuracy of the budget forecasts. Theil's inequality coefficient is based on the MSE $\left(U_{1}\right)$. The forecast error of Theil (1958) is defined as:

$$
\mathbf{U}_{\mathbf{1}}=\frac{\sqrt{1 / n \sum\left(P_{t}-A_{t}\right)^{2}}}{\sqrt{1 / n \sum P_{t}^{2}}+\sqrt{1 / n \sum A_{t}^{2}}}
$$

Where $\mathrm{U}_{1}=$ inequality coefficient, $\mathrm{P}_{\mathrm{t}}=$ predicted value, $\mathrm{A}_{\mathrm{t}}=$ actual value, and $\mathrm{n}=$ the number of years. 
This inequality coefficient ranges from zero to one. When $\mathrm{P}_{t}=\mathrm{A}_{t}$ for all observations (a perfect forecast), $\mathrm{U}_{1}$ equals zero. ${ }^{3}$

$\mathrm{U}_{1}$ has been decomposed in order to indicate systematic and random sources of error. The systematic component is further divided into the proportion of the total forecast error due to bias and the proportion of total forecast error attributable to unequal variation. The derivation of equation (2) is given in detail in Davis (1980).

$$
\mathbf{1}=\frac{\overline{(P}-\bar{A})^{2}}{1 / n \sum\left(P_{t}-A_{t}\right)^{2}}+\frac{(S p-S a)^{2}}{1 / n \sum\left(P_{t}-A_{t}\right)^{2}}+\frac{2(1-r) S p \cdot S a}{1 / n \sum\left(P_{t}-A_{t}\right)^{2}}
$$

In equation (2), $\mathrm{P}$ and $\mathrm{A}$ are mean predicted and mean actual changes, respectively; $\mathrm{Sp}$ and $\mathrm{Sa}$ are the standard deviations of predicted and actual values, respectively; and $r$ is the coefficient of correlation between predicted and actual values.

The first expression of right-hand side (RHS) in equation (2) is the proportion of the total forecast error due to bias. It represents a measure of the proportion of error due to overprediction or underprediction of the average value. The second expression of the RHS in equation (2) is the proportion of total forecast error attributable to unequal variation. In other words, it measures the proportion of error due to overprediction or underprediction of the variance of the values. The

\footnotetext{
${ }^{3}$ Theil's second equation for the inequality coefficient uses a revised measure of forecast error. Theil's $(1966,1971)$ revised measure of inequality is as follows:

$$
\mathbf{U}_{\mathbf{2}}=\frac{\sqrt{1 / n \sum\left(P_{t}-A_{t}\right)^{2}}}{\sqrt{1 / n \sum A_{t}{ }^{2}}}
$$

This measure has the advantage that the denominator does not contain $\mathrm{P}$ and the inequality coefficient does not depend on the forecast. In a perfect forecast, $\mathrm{U}_{2}$ equals to zero. $\mathrm{U}_{2}$ does not have an upper bound.

A more rigorous measure of Theil's inequality statistics is also used by incorporating the lags in the actuals and the difference of the predicted value from the lag of the actuals to capture the magnitude of error:
}

$$
\mathbf{U}_{\mathbf{3}}=\sqrt{\frac{1 / n \sum[P t-a t]^{2}}{1 / n \sum[P t]^{2}+1 / n \sum[a t]^{2}}}
$$

where $a=A_{t}-A_{t-1}, P_{t}=P_{t}-A_{t-1}$, and $n=$ number of years 
third expression of the RHS in equation (2) measures the proportion of forecasting error due to random variation.

The first two sources of error are systematic; presumably they can be reduced by improved forecasting techniques, while the random component is beyond the control of the forecaster (Intriligator 1978; Pindyck and Rubenfield 1998; Theil 1958).

\section{Magnitude of Forecasting Errors}

Our analysis showed that in 28 states the overestimation of revenue receipts amounts to 1.18 percent of gross state domestic product (GSDP) with respect to the forecast deviation between $\mathrm{BE}$ and actuals. The same ratio is slightly reduced to 1.03 percent for RE and actuals. The underestimation (negative deviations of $\mathrm{BE}$ and actuals) of revenue is however negligible (table $1)$.

The state's own tax revenue alone showed 0.40 percent overestimation as a percent of GSDP for all states with regard to forecast errors between $\mathrm{BE}$ and actuals. The errors reduced to 0.22 percent of GSDP for RE versus actuals. The state's own nontax revenue was cumulatively overestimated by 0.11 percent, while the central government's transfers were overestimated to 0.14 percent by GSDP. It would be interesting to analyze the reasons for these forecast errors in the central government's transfers to all states. The design of cess and surcharges is an additional dimension for the reduction in the divisible tax pool transferred from the central government to the states. The cumulative forecast error/deviation between BE and actuals was relatively higher for grants than tax transfers to all states, at 0.66 percent for BE versus actuals (table 1).

The cumulative overestimation of revenue expenditure of all states over the period 2011-16 was 1.05 percent of GSDP with respect to the forecast errors between BE and actuals (table 2). Within the revenue expenditure, the overestimation of social services $(0.53$ percent with respect to RE versus actuals) is higher than economic services and general services. This is broadly giving an indication that against the backdrop of fiscal rules at subnational level, expenditure compression happens more with social sector spending. The cumulative overestimation of general services is 0.34 percent for BE versus actuals and at 0.17 percent for RE versus actuals. 
Table 1: Deviation between BE/RE and Actuals in Revenue Receipts (as a percent of all GSDP, 2011-16)

\begin{tabular}{|c|c|c|c|c|c|c|c|c|c|c|}
\hline & \multicolumn{2}{|c|}{$\begin{array}{l}\text { Revenue } \\
\text { Receipts }\end{array}$} & \multicolumn{2}{|c|}{$\begin{array}{l}\text { States' Own Tax } \\
\text { Revenue }\end{array}$} & \multicolumn{2}{|c|}{$\begin{array}{c}\text { State's Own } \\
\text { Nontax Revenue }\end{array}$} & \multicolumn{2}{|c|}{$\begin{array}{c}\text { Share in Central } \\
\text { Taxes }\end{array}$} & \multicolumn{2}{|c|}{$\begin{array}{l}\text { Grants from } \\
\text { Center }\end{array}$} \\
\hline & $\begin{array}{c}\text { BE- } \\
\text { Actuals }\end{array}$ & $\begin{array}{c}\text { RE- } \\
\text { Actuals }\end{array}$ & $\begin{array}{c}\text { BE- } \\
\text { Actuals }\end{array}$ & $\begin{array}{c}\text { RE- } \\
\text { Actuals }\end{array}$ & $\begin{array}{c}\text { BE- } \\
\text { Actuals }\end{array}$ & $\begin{array}{c}\text { RE- } \\
\text { Actuals }\end{array}$ & $\begin{array}{c}\text { BE- } \\
\text { Actuals }\end{array}$ & $\begin{array}{c}\text { RE- } \\
\text { Actuals }\end{array}$ & $\begin{array}{c}\text { BE- } \\
\text { Actuals }\end{array}$ & $\begin{array}{c}\text { RE- } \\
\text { Actuals }\end{array}$ \\
\hline Overe & 1.20 & 1.05 & 0.40 & 0.22 & 0.11 & 0.08 & 0.14 & 0.12 & 0.67 & 0.66 \\
\hline Underestimation & 0.00 & -0.08 & -0.04 & -0.07 & -0.04 & -0.03 & -0.02 & -0.02 & -0.02 & 0.00 \\
\hline
\end{tabular}

Source: Finance Accounts of States and State Budget documents (various years)

Table 2: Deviation between BE/RE and Actuals in Revenue Expenditure (as percent of all GSDP, 2011-16)

\begin{tabular}{|l|c|c|c|r|r|r|r|r|}
\hline & \multicolumn{2}{c}{$\begin{array}{c}\text { Revenue Expenditure } \\
\text { (total) }\end{array}$} & \multicolumn{2}{c|}{ Social Services } & \multicolumn{2}{c|}{ Economic Services } & \multicolumn{2}{c|}{ General Services } \\
\hline & $\begin{array}{c}\text { BE- } \\
\text { Actuals }\end{array}$ & $\begin{array}{c}\text { RE- } \\
\text { Actuals }\end{array}$ & \multicolumn{1}{c|}{$\begin{array}{c}\text { BE- } \\
\text { Actuals }\end{array}$} & $\begin{array}{c}\text { RE- } \\
\text { Actuals }\end{array}$ & $\begin{array}{c}\text { BE- } \\
\text { Actuals }\end{array}$ & $\begin{array}{c}\text { RE- } \\
\text { Actuals }\end{array}$ & $\begin{array}{c}\text { BE- } \\
\text { Actuals }\end{array}$ & $\begin{array}{c}\text { RE- } \\
\text { Actuals }\end{array}$ \\
\hline Overestimation & 1.05 & 1.29 & 0.37 & 0.53 & 0.19 & 0.39 & 0.34 & 0.17 \\
\hline Underestimation & -0.01 & -0.10 & -0.02 & -0.05 & -0.05 & -0.02 & -0.03 & -0.05 \\
\hline
\end{tabular}

Source: Finance Accounts of States and State Budget documents (various years)

Table 3: Deviation between BE/RE and Actuals in Capital Expenditure (as percent of all GSDP, 2011-16)

\begin{tabular}{|c|c|c|c|c|c|c|c|c|}
\hline 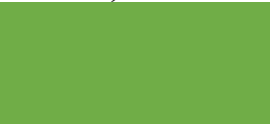 & \multicolumn{2}{|c|}{$\begin{array}{l}\text { Capital } \\
\text { Expenditure } \\
\text { (total) }\end{array}$} & \multicolumn{2}{|c|}{ Social Services } & \multicolumn{2}{|c|}{$\begin{array}{c}\text { Economic } \\
\text { Services }\end{array}$} & \multicolumn{2}{|c|}{ General Services } \\
\hline & $\begin{array}{c}\text { BE- } \\
\text { Actuals }\end{array}$ & $\begin{array}{c}\text { RE- } \\
\text { Actuals }\end{array}$ & $\begin{array}{c}\text { BE- } \\
\text { Actuals }\end{array}$ & $\begin{array}{c}\text { RE- } \\
\text { Actuals }\end{array}$ & $\begin{array}{c}\text { BE- } \\
\text { Actuals }\end{array}$ & $\begin{array}{c}\text { RE- } \\
\text { Actuals }\end{array}$ & $\begin{array}{c}\text { BE- } \\
\text { Actuals }\end{array}$ & $\begin{array}{c}\text { RE- } \\
\text { Actuals }\end{array}$ \\
\hline Overestimation & 0.39 & 0.38 & 0.15 & 0.14 & 0.20 & 0.19 & 0.08 & 0.06 \\
\hline Underestimation & -0.02 & -0.01 & 0.00 & 0.00 & -0.05 & -0.02 & 0.00 & 0.00 \\
\hline
\end{tabular}

Source: Finance Accounts of States and State Budget documents (various years)

The cumulative overestimation of capital expenditure in the period under analysis was as high as 0.38 percent of GSDP for both BE-actuals and RE-actuals (table 3).

Mean Error (ME) and Root of Mean Error Square (RMSE)

Analyzing the ME and RMSE, we find that the values of MSE and RMSE (as a proportion of actuals) seem to be higher in the case of capital expenditure compared to revenue expenditure. This is true of almost all of states. Only in the cases of Haryana, Karnataka, and Odisha is the MSE (as a proportion of actuals) higher for revenue expenditure compared to capital expenditure (table 4). Furthermore, only in Himachal, Karnataka, Kerala, and Uttarakhand is the RMSE as proportion of actuals higher in the case of revenue expenditure compared to capital expenditure. Secondly, the MSE and RMSE (as a proportion of actuals) are higher in the case of nontax 
revenue (including grants) compared to tax revenue (including tax transfers) at the all-state level. The all-state ME as a proportion of actuals for total tax revenue is 0.0496 and the all-state ME for nontax revenues as a proportion of actuals is 0.2049 . Similarly, in the case of RMSE, the allstate RMSE as a proportion of actuals for total tax revenue is 548.09 and the same for nontax revenue is 1140.79 . The main reason the ME and RMSE are higher for nontax revenue is because the value of these two indicators is very high for the grants from the central government. The RMSE as a proportion of actuals for grants from the central government is 1157.62 and the RMSE for states' own nontax revenue is only 372.02. Similarly, the states' ME for own tax revenue is only 0.0544 , whereas it is 0.282 for grants from the central government. 
Table 4: Mean Error (ME) and Root Mean Square Error (RMSE): Budget Estimates (BE) and Actuals

\begin{tabular}{|c|c|c|c|c|c|c|c|c|c|}
\hline States & $\begin{array}{c}\text { State Own } \\
\text { Tax } \\
\text { Revenue }\end{array}$ & $\begin{array}{c}\text { Share in } \\
\text { Central } \\
\text { Taxes }\end{array}$ & $\begin{array}{c}\text { States Own } \\
\text { Nontax } \\
\text { Revenue }\end{array}$ & $\begin{array}{l}\text { Grants } \\
\text { from } \\
\text { Center }\end{array}$ & $\begin{array}{c}\text { Revenue } \\
\text { Expenditure }\end{array}$ & $\begin{array}{c}\text { Capital } \\
\text { Expenditure }\end{array}$ & $\begin{array}{l}\text { Revenue } \\
\text { Deficit }\end{array}$ & $\begin{array}{l}\text { Fiscal } \\
\text { Deficit }\end{array}$ & $\begin{array}{l}\text { Primary } \\
\text { Deficit }\end{array}$ \\
\hline \multirow[t]{2}{*}{ Andhra Pradesh } & 0.04 & 0.04 & -0.07 & 0.14 & 0.02 & 0.07 & -0.45 & -0.04 & -0.11 \\
\hline & 0.02 & 0.01 & 0.03 & 0.07 & 0.02 & 0.05 & -0.17 & -0.08 & -0.16 \\
\hline \multirow[t]{2}{*}{ Arunachal Pradesh } & 0.08 & 0.01 & 0.22 & 0.11 & 0.12 & 0.75 & -0.07 & 3.19 & 13.61 \\
\hline & 0.04 & 0.00 & 0.05 & 0.06 & 0.02 & 0.17 & 0.27 & -0.91 & -3.98 \\
\hline \multirow[t]{2}{*}{ Assam } & 0.07 & 0.05 & 0.10 & 0.51 & 0.33 & 1.56 & 1.42 & 0.64 & 0.93 \\
\hline & 0.02 & 0.01 & 0.03 & 0.12 & 0.05 & 0.24 & -0.50 & -0.23 & -0.34 \\
\hline \multirow[t]{2}{*}{ Bihar } & 0.07 & 0.04 & 0.27 & 0.37 & 0.27 & 0.34 & -1.52 & 1.18 & 2.39 \\
\hline & 0.02 & 0.01 & 0.10 & 0.08 & 0.05 & 0.07 & 0.46 & -0.27 & -0.55 \\
\hline \multirow[t]{2}{*}{ Chhattisgarh } & 0.11 & 0.06 & 0.26 & 0.54 & 0.17 & 0.31 & 0.96 & 0.19 & 0.26 \\
\hline & 0.02 & 0.01 & 0.03 & 0.10 & 0.03 & 0.05 & 0.38 & -0.13 & -0.20 \\
\hline \multirow[t]{2}{*}{ Goa } & 0.03 & 0.03 & 0.02 & 0.61 & 0.09 & 0.67 & -8.79 & 0.90 & 4.02 \\
\hline & 0.01 & 0.01 & 0.01 & 0.10 & 0.02 & 0.10 & 2.19 & -0.25 & -1.07 \\
\hline \multirow[t]{2}{*}{ Gujarat } & 0.00 & 0.07 & 0.04 & 0.35 & 0.04 & 0.06 & 0.23 & 0.00 & 0.00 \\
\hline & 0.01 & 0.01 & 0.01 & 0.07 & 0.01 & 0.02 & 0.12 & -0.03 & -0.12 \\
\hline \multirow[t]{2}{*}{ Haryana } & 0.06 & 0.04 & 0.07 & 0.54 & 0.11 & 0.04 & 0.06 & 0.26 & 0.49 \\
\hline & 0.01 & 0.01 & 0.02 & 0.11 & 0.02 & 0.05 & -0.05 & -0.11 & -0.21 \\
\hline \multirow[t]{2}{*}{ Himachal Pradesh } & -0.01 & 0.12 & -0.02 & 0.08 & 0.07 & 0.07 & 0.87 & 0.02 & 0.77 \\
\hline & 0.01 & 0.03 & 0.05 & 0.02 & 0.01 & 0.01 & -0.23 & -0.03 & -0.35 \\
\hline \multirow[t]{2}{*}{ Jammu and Kashmir } & 0.05 & 0.04 & 0.14 & 0.19 & 0.01 & 0.56 & 7.68 & -0.05 & -0.16 \\
\hline & 0.01 & 0.01 & 0.05 & 0.03 & 0.01 & 0.10 & 1.38 & -0.29 & -0.89 \\
\hline \multirow[t]{2}{*}{ Jharkhand } & 0.15 & 0.04 & 0.19 & 0.86 & 0.22 & 0.31 & 0.81 & 0.09 & 0.20 \\
\hline & 0.03 & 0.01 & 0.04 & 0.18 & 0.04 & 0.07 & 0.25 & -0.10 & -0.21 \\
\hline \multirow[t]{2}{*}{ Karnataka } & -0.02 & 0.02 & -0.06 & 0.34 & 0.25 & -0.02 & -0.43 & 0.03 & 0.06 \\
\hline & 0.00 & 0.01 & 0.02 & 0.09 & 0.10 & 0.01 & 0.15 & -0.03 & -0.05 \\
\hline \multirow[t]{2}{*}{ Kerala } & 0.06 & 0.04 & 0.05 & 0.24 & 0.16 & 0.18 & 0.60 & 0.43 & 0.92 \\
\hline & 0.01 & 0.01 & 0.01 & 0.06 & 0.07 & 0.06 & -0.36 & -0.24 & -0.50 \\
\hline \multirow[t]{2}{*}{ Madhya Pradesh } & 0.01 & 0.05 & 0.02 & 0.29 & 0.10 & 0.07 & -0.21 & 0.28 & 0.71 \\
\hline & 0.01 & 0.01 & 0.01 & 0.08 & 0.02 & 0.02 & 0.04 & -0.08 & -0.20 \\
\hline \multirow[t]{2}{*}{ Maharashtra } & 0.01 & 0.00 & 0.12 & 0.40 & 0.06 & 0.14 & 0.37 & 0.18 & 1.71 \\
\hline & 0.00 & 0.00 & 0.02 & 0.08 & 0.01 & 0.02 & -0.15 & -0.07 & -0.64 \\
\hline \multirow[t]{2}{*}{ Manipur } & 0.08 & 0.04 & 0.30 & 0.15 & 0.15 & 0.33 & -0.02 & 1.41 & -4.61 \\
\hline & 0.03 & 0.01 & 0.09 & 0.04 & 0.03 & 0.06 & 0.05 & -0.32 & 1.05 \\
\hline
\end{tabular}




\begin{tabular}{|c|c|c|c|c|c|c|c|c|c|}
\hline States & $\begin{array}{l}\text { State Own Tax } \\
\text { Revenue }\end{array}$ & $\begin{array}{c}\text { Share in } \\
\text { Central Taxes }\end{array}$ & $\begin{array}{c}\text { States Own } \\
\text { Nontax Revenue }\end{array}$ & $\begin{array}{l}\text { Grants from } \\
\text { Center }\end{array}$ & $\begin{array}{c}\text { Revenue } \\
\text { Expenditure }\end{array}$ & $\begin{array}{c}\text { Capital } \\
\text { Expenditure }\end{array}$ & $\begin{array}{l}\text { Revenue } \\
\text { Deficit }\end{array}$ & Fiscal Deficit & $\begin{array}{l}\text { Primary } \\
\text { Deficit }\end{array}$ \\
\hline \multirow[t]{2}{*}{ Meghalaya } & -0.02 & 0.06 & 0.17 & 0.66 & 0.28 & 0.47 & 1.50 & -0.20 & -0.44 \\
\hline & 0.03 & 0.01 & 0.08 & 0.14 & 0.07 & 0.09 & 0.34 & -0.17 & -0.28 \\
\hline \multirow[t]{2}{*}{ Mizoram } & -0.05 & -0.01 & 0.09 & 0.15 & 0.14 & 0.40 & -0.88 & 0.90 & 2.31 \\
\hline & 0.01 & 0.01 & 0.04 & 0.03 & 0.03 & 0.08 & 0.54 & -0.40 & -0.97 \\
\hline \multirow{2}{*}{ Nagaland } & -0.05 & 0.00 & -0.11 & 0.09 & 0.14 & 0.29 & -0.27 & 1.10 & -20.32 \\
\hline & 0.02 & 0.00 & 0.04 & 0.01 & 0.03 & 0.06 & 0.08 & -0.32 & 5.69 \\
\hline \multirow[t]{2}{*}{ Orissa } & -0.01 & 0.02 & -0.10 & 0.39 & 0.12 & 0.03 & -0.40 & 0.80 & 2.71 \\
\hline & 0.00 & 0.01 & 0.03 & 0.08 & 0.02 & 0.01 & 0.09 & -0.22 & -1.12 \\
\hline \multirow[t]{2}{*}{ Punjab } & 0.09 & 0.03 & 0.51 & 0.37 & 0.08 & 0.77 & -0.20 & -0.49 & -1.76 \\
\hline & 0.02 & 0.01 & 0.12 & 0.10 & 0.02 & 0.17 & -0.04 & -0.18 & -0.67 \\
\hline \multirow[t]{2}{*}{ Rajasthan } & 0.02 & 0.00 & 0.01 & 0.17 & 0.06 & 0.12 & 2.16 & 0.18 & 0.31 \\
\hline & 0.01 & 0.00 & 0.01 & 0.04 & 0.01 & 0.03 & -0.63 & -0.04 & -0.07 \\
\hline \multirow[t]{2}{*}{ Sikkim } & -0.07 & 0.04 & 0.27 & 0.47 & 0.19 & 0.71 & 0.67 & 0.76 & -127.83 \\
\hline & 0.02 & 0.01 & 0.07 & 0.12 & 0.04 & 0.15 & 0.13 & -0.29 & 34.27 \\
\hline \multirow[t]{2}{*}{ Tamil Nadu } & 0.07 & 0.02 & -0.02 & 0.10 & 0.05 & 0.14 & -0.31 & 0.03 & 0.07 \\
\hline & 0.02 & 0.01 & 0.02 & 0.02 & 0.01 & 0.03 & -0.10 & -0.02 & -0.06 \\
\hline \multirow[t]{2}{*}{ Tripura } & 0.04 & 0.03 & 0.03 & 0.15 & 0.11 & 0.30 & 0.06 & 1.26 & -2.47 \\
\hline & 0.02 & 0.01 & 0.03 & 0.03 & 0.02 & 0.05 & 0.04 & -0.45 & 0.66 \\
\hline \multirow[t]{2}{*}{ Uttar Pradesh } & 0.03 & 0.05 & 0.07 & 0.33 & 0.08 & 0.10 & 0.19 & 0.06 & 0.18 \\
\hline & 0.01 & 0.01 & 0.03 & 0.09 & 0.01 & 0.02 & 0.08 & -0.03 & -0.08 \\
\hline \multirow[t]{2}{*}{ Uttarakhand } & -0.85 & -0.48 & -0.82 & 0.06 & -0.60 & -0.27 & 9.86 & -0.74 & -0.79 \\
\hline & 0.15 & 0.09 & 0.16 & 0.01 & 0.11 & 0.06 & 1.84 & -0.20 & -0.26 \\
\hline \multirow[t]{2}{*}{ West Bengal } & 0.02 & 0.04 & 0.22 & 0.13 & 0.02 & 0.34 & -0.15 & 0.03 & 0.27 \\
\hline & 0.01 & 0.01 & 0.11 & 0.04 & 0.01 & 0.06 & -0.06 & -0.03 & -0.28 \\
\hline
\end{tabular}

Note: The first figure is ME and the second figure is RMSE.

Source: Comptroller and Auditor General (CAG) office finance accounts of states and state budget documents (basic data; various years) 
Table 5: Mean Error (ME) and Root Mean Square Error (RMSE): Revised Estimates (RE) and Actuals

\begin{tabular}{|c|c|c|c|c|c|c|c|c|c|}
\hline States & $\begin{array}{c}\text { State Own } \\
\text { Tax } \\
\text { Revenue }\end{array}$ & $\begin{array}{c}\text { Share in } \\
\text { Central } \\
\text { Taxes }\end{array}$ & $\begin{array}{l}\text { States Own } \\
\text { Nontax } \\
\text { Revenue }\end{array}$ & $\begin{array}{l}\text { Grants } \\
\text { from } \\
\text { Center }\end{array}$ & $\begin{array}{c}\text { Revenue } \\
\text { Expenditure }\end{array}$ & $\begin{array}{c}\text { Capital } \\
\text { Expenditure }\end{array}$ & $\begin{array}{c}\text { Revenue } \\
\text { Deficit }\end{array}$ & $\begin{array}{l}\text { Fiscal } \\
\text { Deficit }\end{array}$ & $\begin{array}{c}\text { Primary } \\
\text { Deficit }\end{array}$ \\
\hline \multirow[t]{2}{*}{ Andhra Pradesh } & 0.01 & 0.01 & -0.01 & 0.03 & 0.00 & 0.01 & -0.09 & -0.01 & -0.02 \\
\hline & 0.04 & 0.03 & 0.06 & 0.15 & 0.05 & 0.12 & -0.40 & -0.14 & -0.27 \\
\hline \multirow{2}{*}{$\begin{array}{l}\text { Arunachal } \\
\text { Pradesh }\end{array}$} & 0.02 & 0.00 & 0.04 & 0.02 & 0.02 & 0.15 & -0.01 & 0.64 & 2.72 \\
\hline & 0.08 & 0.00 & 0.13 & 0.13 & 0.06 & 0.36 & 0.56 & -2.03 & -8.84 \\
\hline \multirow[t]{2}{*}{ Assam } & 0.01 & 0.01 & 0.02 & 0.10 & 0.07 & 0.31 & 0.28 & 0.13 & 0.19 \\
\hline & 0.05 & 0.03 & 0.07 & 0.26 & 0.17 & 0.79 & -1.27 & -0.30 & -0.44 \\
\hline \multirow[t]{2}{*}{ Bihar } & 0.01 & 0.01 & 0.05 & 0.07 & 0.05 & 0.07 & -0.30 & 0.24 & 0.48 \\
\hline & 0.05 & 0.02 & 0.22 & 0.18 & 0.13 & 0.17 & 1.07 & -0.58 & -1.18 \\
\hline \multirow[t]{2}{*}{ Chhattisgarh } & 0.02 & 0.01 & 0.05 & 0.11 & 0.03 & 0.06 & 0.19 & 0.04 & 0.05 \\
\hline & 0.07 & 0.03 & 0.16 & 0.27 & 0.09 & 0.15 & 0.81 & -0.20 & -0.31 \\
\hline \multirow[t]{2}{*}{ Goa } & 0.01 & 0.01 & 0.00 & 0.12 & 0.02 & 0.13 & -1.76 & 0.18 & 0.80 \\
\hline & 0.02 & 0.02 & 0.02 & 0.34 & 0.04 & 0.32 & 4.70 & -0.42 & -1.84 \\
\hline \multirow[t]{2}{*}{ Gujarat } & 0.00 & 0.01 & 0.01 & 0.07 & 0.01 & 0.01 & 0.05 & 0.00 & 0.00 \\
\hline & 0.02 & 0.03 & 0.05 & 0.19 & 0.02 & 0.04 & 0.27 & -0.04 & -0.18 \\
\hline \multirow[t]{2}{*}{ Haryana } & 0.01 & 0.01 & 0.01 & 0.11 & 0.02 & 0.01 & 0.01 & 0.05 & 0.10 \\
\hline & 0.04 & 0.02 & 0.05 & 0.25 & 0.05 & 0.11 & -0.10 & -0.23 & -0.45 \\
\hline \multirow[t]{2}{*}{ Himachal Pradesh } & 0.00 & 0.02 & 0.00 & 0.02 & 0.01 & 0.01 & 0.17 & 0.00 & 0.15 \\
\hline & 0.02 & 0.07 & 0.11 & 0.05 & 0.04 & 0.04 & -0.66 & -0.03 & -0.74 \\
\hline \multirow{2}{*}{$\begin{array}{l}\text { Jammu and } \\
\text { Kashmir }\end{array}$} & 0.01 & 0.01 & 0.03 & 0.04 & 0.00 & 0.11 & 1.54 & -0.01 & -0.03 \\
\hline & 0.03 & 0.02 & 0.12 & 0.10 & 0.01 & 0.28 & 3.68 & -0.09 & -0.23 \\
\hline \multirow[t]{2}{*}{ Jharkhand } & 0.03 & 0.01 & 0.04 & 0.17 & 0.04 & 0.06 & 0.16 & 0.02 & 0.04 \\
\hline & 0.09 & 0.02 & 0.09 & 0.41 & 0.10 & 0.15 & 0.56 & -0.14 & -0.29 \\
\hline \multirow[t]{2}{*}{ Karnataka } & 0.00 & 0.00 & -0.01 & 0.07 & 0.05 & 0.00 & -0.09 & 0.01 & 0.01 \\
\hline & 0.01 & 0.02 & 0.04 & 0.21 & 0.22 & 0.02 & 0.22 & -0.02 & -0.04 \\
\hline \multirow[t]{2}{*}{ Kerala } & 0.01 & 0.01 & 0.01 & 0.05 & 0.03 & 0.04 & 0.12 & 0.09 & 0.18 \\
\hline & 0.03 & 0.02 & 0.03 & 0.12 & 0.15 & 0.14 & -0.81 & -0.53 & -1.12 \\
\hline \multirow[t]{2}{*}{ Madhya Pradesh } & 0.00 & 0.01 & 0.00 & 0.06 & 0.02 & 0.01 & -0.04 & 0.06 & 0.14 \\
\hline & 0.02 & 0.03 & 0.04 & 0.17 & 0.05 & 0.05 & 0.17 & -0.15 & -0.40 \\
\hline \multirow[t]{2}{*}{ Maharashtra } & 0.00 & 0.00 & 0.02 & 0.08 & 0.01 & 0.03 & 0.07 & 0.04 & 0.34 \\
\hline & 0.01 & 0.00 & 0.05 & 0.20 & 0.03 & 0.08 & -0.31 & -0.10 & -0.96 \\
\hline \multirow[t]{2}{*}{ Manipur } & 0.02 & 0.01 & 0.06 & 0.03 & 0.03 & 0.07 & 0.00 & 0.28 & -0.92 \\
\hline & 0.07 & 0.03 & 0.20 & 0.08 & 0.07 & 0.15 & 0.12 & -0.71 & 2.30 \\
\hline \multirow[t]{2}{*}{ Meghalaya } & 0.00 & 0.01 & 0.03 & 0.13 & 0.06 & 0.09 & 0.30 & -0.04 & -0.09 \\
\hline & 0.07 & 0.03 & 0.18 & 0.33 & 0.16 & 0.23 & 0.77 & -0.25 & -0.55 \\
\hline \multirow[t]{2}{*}{ Mizoram } & -0.01 & 0.00 & 0.02 & 0.03 & 0.03 & 0.08 & -0.18 & 0.18 & 0.46 \\
\hline & 0.04 & 0.02 & 0.09 & 0.08 & 0.07 & 0.20 & 1.14 & -0.56 & -1.44 \\
\hline \multirow[t]{2}{*}{ Nagaland } & -0.01 & 0.00 & -0.02 & 0.02 & 0.03 & 0.06 & -0.05 & 0.22 & -4.06 \\
\hline & 0.04 & 0.00 & 0.09 & 0.05 & 0.08 & 0.15 & 0.18 & -0.52 & 9.73 \\
\hline Orissa & 0.00 & 0.00 & -0.02 & 0.08 & 0.02 & 0.01 & -0.08 & 0.16 & 0.54 \\
\hline & 0.01 & 0.02 & 0.06 & 0.19 & 0.06 & 0.03 & 0.18 & -0.37 & -1.67 \\
\hline Punjab & 0.09 & 0.03 & 0.51 & 0.37 & 0.08 & 0.77 & -0.20 & -0.49 & -1.76 \\
\hline & 0.04 & 0.03 & 0.28 & 0.21 & 0.04 & 0.37 & -0.10 & -0.41 & -1.49 \\
\hline Rajasthan & 0.00 & 0.00 & 0.00 & 0.03 & 0.01 & 0.02 & 0.43 & 0.04 & 0.06 \\
\hline & 0.02 & 0.00 & 0.02 & 0.09 & 0.03 & 0.06 & -1.29 & -0.08 & -0.14 \\
\hline Sikkim & -0.01 & 0.01 & 0.05 & 0.09 & 0.04 & 0.14 & 0.13 & 0.15 & -25.57 \\
\hline & 0.05 & 0.03 & 0.15 & 0.22 & 0.09 & 0.32 & 0.31 & -0.37 & 62.99 \\
\hline Tamil Nadu & 0.01 & 0.00 & 0.00 & 0.02 & 0.01 & 0.03 & -0.06 & 0.01 & 0.01 \\
\hline & 0.04 & 0.01 & 0.04 & 0.07 & 0.02 & 0.07 & -0.27 & -0.03 & -0.07 \\
\hline Tripura & 0.01 & 0.01 & 0.01 & 0.03 & 0.02 & 0.06 & 0.01 & 0.25 & -0.49 \\
\hline & 0.04 & 0.03 & 0.06 & 0.08 & 0.06 & 0.14 & 0.12 & -0.65 & 1.33 \\
\hline
\end{tabular}




\begin{tabular}{|c|c|c|c|c|c|c|c|c|c|}
\hline States & $\begin{array}{c}\text { State Own } \\
\text { Tax } \\
\text { Revenue }\end{array}$ & $\begin{array}{l}\text { Share in } \\
\text { Central } \\
\text { Taxes }\end{array}$ & $\begin{array}{c}\text { States Own } \\
\text { Nontax } \\
\text { Revenue }\end{array}$ & $\begin{array}{l}\text { Grants } \\
\text { from } \\
\text { Center }\end{array}$ & $\begin{array}{c}\text { Revenue } \\
\text { Expenditure }\end{array}$ & $\begin{array}{c}\text { Capital } \\
\text { Expenditure }\end{array}$ & $\begin{array}{c}\text { Revenue } \\
\text { Deficit }\end{array}$ & $\begin{array}{l}\text { Fiscal } \\
\text { Deficit }\end{array}$ & $\begin{array}{l}\text { Primary } \\
\text { Deficit }\end{array}$ \\
\hline \multirow[t]{2}{*}{ Uttar Pradesh } & 0.01 & 0.01 & 0.01 & 0.07 & 0.02 & 0.02 & 0.04 & 0.01 & 0.04 \\
\hline & 0.02 & 0.03 & 0.06 & 0.18 & 0.04 & 0.06 & 0.20 & -0.06 & -0.15 \\
\hline \multirow[t]{2}{*}{ Uttarakhand } & -0.17 & -0.1 & -0.16 & 0.01 & -0.12 & -0.05 & 1.97 & -0.15 & -0.16 \\
\hline & 0.39 & 0.22 & 0.37 & 0.03 & 0.28 & 0.14 & 6.34 & -0.38 & -0.55 \\
\hline \multirow[t]{2}{*}{ West Bengal } & 0.00 & 0.01 & 0.04 & 0.03 & 0.00 & 0.07 & -0.03 & 0.01 & 0.05 \\
\hline & 0.02 & 0.03 & 0.19 & 0.09 & 0.01 & 0.16 & -0.14 & -0.07 & -0.60 \\
\hline
\end{tabular}

Note: The first figure is MSE and the second figure is RMSE.

Source: CAG office finance accounts of states and state budget documents (basic data; various years)

\section{Forecasting Errors: Overestimate or Underestimate?}

One of the limitations of RMSE is that we cannot find the sign of the error. We attempt to calculate fiscal marksmanship indices in this section that will help us assess whether the budgetary estimates are overestimates or underestimates. This ratio would give us information on whether the $\mathrm{BE}$ or $\mathrm{RE}$ is an underestimate or an overestimate. If the value of the ratio is above one, this indicates that, on average, the indicator has been overestimated. Conversely, if the value is below one it can be said that it is an underestimate. In case of $\mathrm{BE}$, it can be observed that most of the categories are overestimated on both the revenue and the expenditure sides.

Table 6: Descriptive Statistics of Fiscal Marksmanship Ratio: Revenue and its Components (BE-Actuals)

\begin{tabular}{|c|c|c|c|c|c|c|c|}
\hline & $\begin{array}{c}\text { Total } \\
\text { Revenue } \\
\text { Receipt }\end{array}$ & $\begin{array}{c}\text { Tax } \\
\text { Revenue }\end{array}$ & $\begin{array}{c}\text { States } \\
\text { Own Tax } \\
\text { Revenue }\end{array}$ & $\begin{array}{c}\text { Share in } \\
\text { Central } \\
\text { Taxes }\end{array}$ & $\begin{array}{l}\text { Nontax } \\
\text { Revenue }\end{array}$ & $\begin{array}{c}\text { States } \\
\text { Own Tax } \\
\text { Revenue }\end{array}$ & $\begin{array}{l}\text { Grants } \\
\text { from } \\
\text { Center }\end{array}$ \\
\hline Median & 1.115 & 1.029 & 1.003 & 1.056 & 1.210 & 1.120 & 1.332 \\
\hline Mean & 1.118 & 1.039 & 1.097 & 1.038 & 1.241 & 1.156 & 1.325 \\
\hline Max & 1.360 & 1.223 & 1.072 & 1.166 & 1.698 & 2.191 & 2.121 \\
\hline Min & 1.012 & 0.916 & 0.952 & 0.866 & 0.839 & 0.828 & 0.803 \\
\hline $\begin{array}{l}\text { Standard } \\
\text { Deviation }\end{array}$ & 0.087 & 0.056 & 0.077 & 0.053 & 0.199 & 0.280 & 0.269 \\
\hline
\end{tabular}

Source: CAG office finance accounts of states and state budget documents (basic data; various years)

On the total revenue receipt, all the states have overestimated the overall revenue, ranging from a maximum value of 1.36 (Meghalaya) and a minimum value of 1.01 (Rajasthan). The median value of total revenue receipt is 1.12 (table 6). Correspondingly, both the tax revenue and nontax revenue are generally overestimated. However, there are a few states where there has been underestimation of tax revenue and nontax revenue. Tax revenue was underestimated in Karnataka and Orissa, and nontax revenues were underestimated 
in Tamil Nadu and Rajasthan. An interesting observation from the data is that the standard deviation of this index for the nontax revenues (for both states' own nontax revenue and grants from the central government) was considerably higher than the tax revenues. In the table 6 , the standard deviation for tax revenue is 0.053 , whereas the standard deviation of nontax revenue is 0.199 . This shows that the ratio of BE-actuals is relatively more spread compared to tax revenues. An observation that is worth noting is that the higher standard deviation of the ratio of $\mathrm{BE}$ to actuals for nontax revenue compared to tax revenue complements the fact that the ME and RMSE also had a similar trend. Coupling the results from this and the previous sections, one can conclude that while the $\mathrm{BE}$ is generally overestimated for both tax revenues and nontax revenues, the errors are generally higher for nontax revenues compared to tax revenues.

Table 7: Descriptive Statistics of Fiscal Marksmanship Ratio: Revenue Expenditure

\begin{tabular}{|l|r|r|r|r|}
\hline & \multicolumn{1}{c}{$\begin{array}{c}\text { Revenue } \\
\text { Expenditure }\end{array}$} & $\begin{array}{c}\text { Social } \\
\text { Services }\end{array}$ & $\begin{array}{c}\text { Economic } \\
\text { Services }\end{array}$ & $\begin{array}{c}\text { Nondevelopmental } \\
\text { Expenditure }\end{array}$ \\
\hline Median & 1.072 & 1.074 & 1.069 & 1.040 \\
\hline Mean & 1.094 & 1.086 & 1.117 & 1.055 \\
\hline Max & 1.279 & 1.432 & 1.715 & 1.319 \\
\hline Min & 0.950 & 0.866 & 0.895 & 0.920 \\
\hline $\begin{array}{l}\text { Standard } \\
\text { Deviation }\end{array}$ & 0.084 & 0.122 & 0.174 & 0.088 \\
\hline
\end{tabular}

Source: CAG office finance accounts of states and state budget documents (basic data; various years)

When we consider the expenditure side, we can observe that it is generally the case that both revenue expenditure and capital expenditure have been overestimated. In the case of revenue expenditure, all of the states except Nagaland and Assam have underestimates. In the case of capital expenditure, all the states besides Karnataka, Uttar Pradesh, and Himachal Pradesh have overestimates. However, one trend that can observed is the range and standard deviation of capital expenditure is much higher compared to revenue expenditure (both overall and component-wise). The maximum and minimum of the revenue expenditure is 1.279 and 0.95 , respectively, for revenue expenditure (table 7). This is considerably lower compared to the maximum and minimum of this index for capital expenditure, which are 2.476 and 0.956 , respectively (table 8 ). 
Table 8: Descriptive Statistics of Fiscal Marksmanship Ratio: Capital Expenditure

\begin{tabular}{|l|r|r|r|r|}
\hline & $\begin{array}{c}\text { Capital } \\
\text { Expenditure }\end{array}$ & Social Services & $\begin{array}{c}\text { Economic } \\
\text { Services }\end{array}$ & $\begin{array}{c}\text { Nondevelopmental } \\
\text { Expenditure }\end{array}$ \\
\hline Median & 1.269 & 1.306 & 1.185 & 1.368 \\
\hline Mean & 1.335 & 1.446 & 1.197 & 1.941 \\
\hline Max & 2.476 & 3.305 & 2.113 & 9.879 \\
\hline Standard Deviation & 0.956 & 0.659 & 0.570 & 0.800 \\
\hline
\end{tabular}

Source: CAG office finance accounts of states and state budget documents (basic data; various years)

The standard deviation for capital expenditure is 0.359 , which is around fourfold higher than the standard deviation of revenue expenditure (table 8). It was observed in the previous section, the MSE and RMSE are higher for capital expenditure compared to revenue expenditure. Since most of the states had overestimates of both the revenue and capital expenditure it can be concluded that the forecasting errors in capital expenditure tend to be higher compared to revenue expenditure.

\section{Statewise Fiscal Marksmanship Ratios of Macro-Fiscal Variables}

For fiscal marksmanship ratios, we divided the BE by the actual values and take the average for the years 2011-16. Therefore, if the values in the figures 1-5 is above one, it suggests that, on average, the indicator has been overestimated. Conversely, if the value is below one it can be said that it is underestimated. In case of BE, it can be observed that most of the categories are overestimated on both the revenue and expenditure side. It can be observed that both the mean and median are over one, indicating most of them are overestimated. When we observe the statewise trend, most of the categories have more than 20 states with an overestimate. One can observe a similar trend in the case of RE. On average, both the revenue and expenditure have been overestimated. In most of the categories, there are overestimations in over 20 states. It is worth noting that while most of the categories have overestimates, in case of revenue deficit, fiscal deficit, and primary deficit, merely 11, 18, and 16 states, respectively, had underestimates. This is a trend similar to the BE. The fiscal marksmanship ratios suggest that forecast errors in grants are greater than other macro-fiscal variables (figure 3). 
Figure 1: Fiscal Marksmanship Ratio of Own Tax Revenue

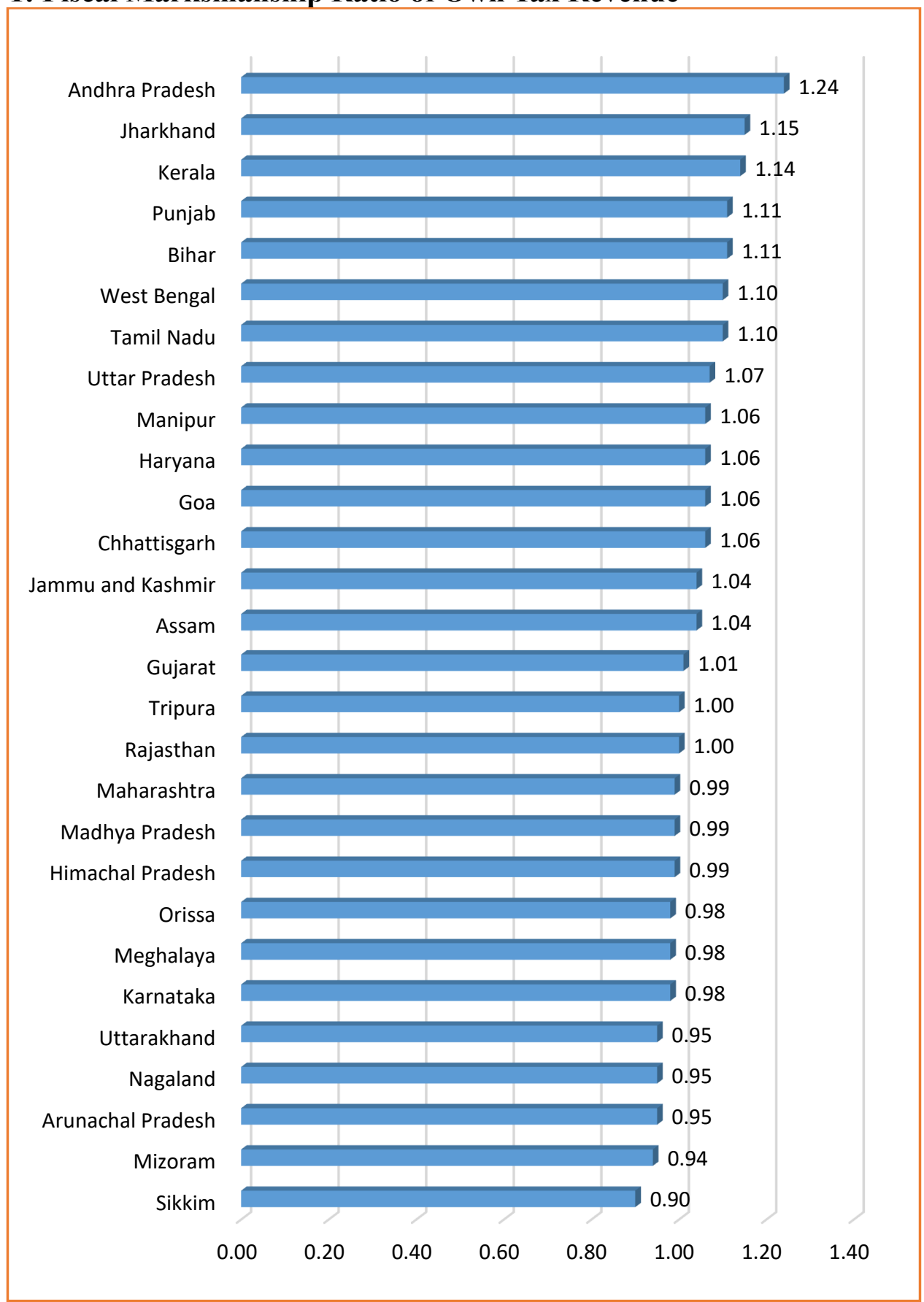

Source: CAG office finance accounts of states and state budget documents (basic data; various years) 
Figure 2: Fiscal Marksmanship Ratio of Tax Transfer

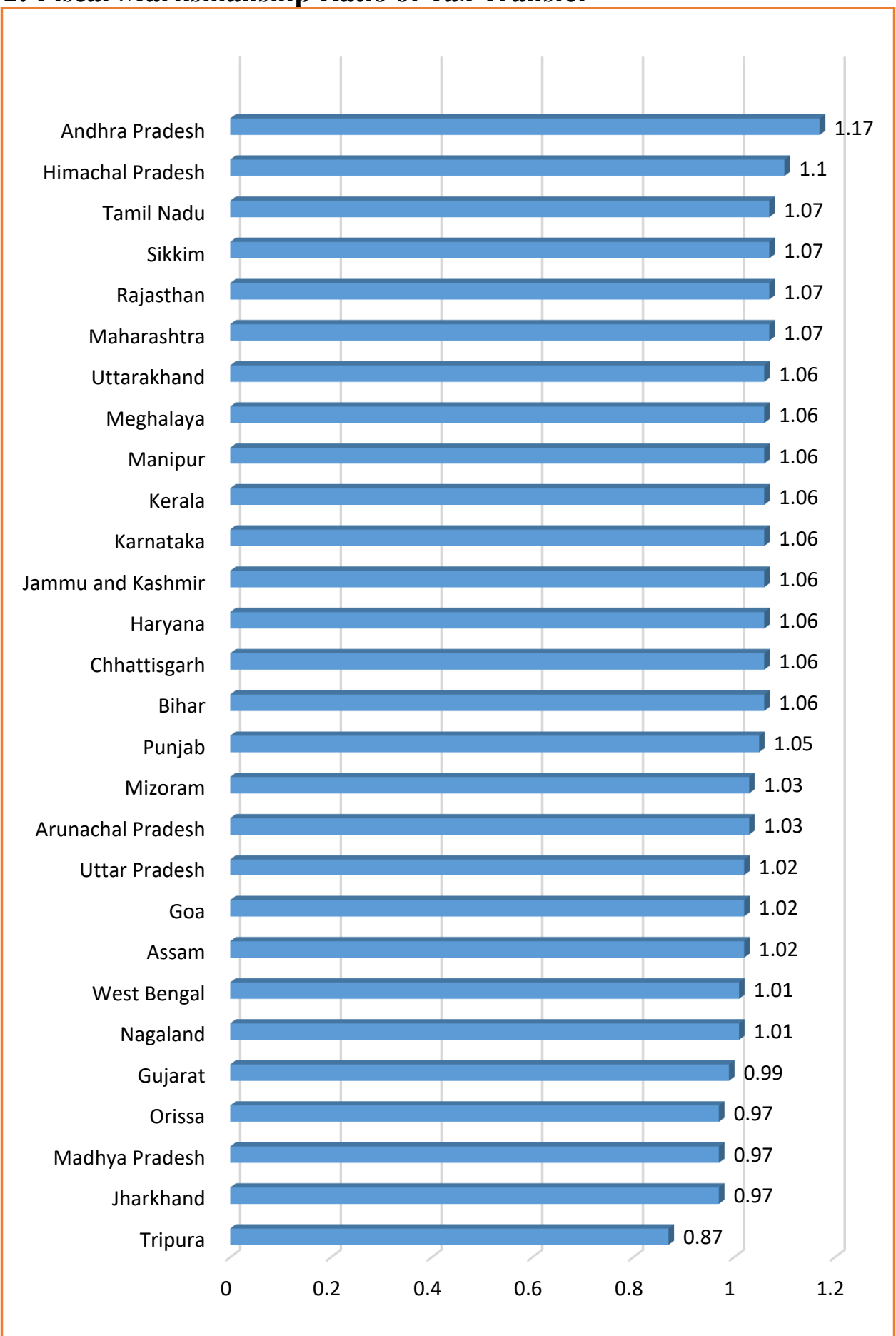

Source: CAG office finance accounts of states and state budget documents (basic data; various years) 
Figure 3: Fiscal Marksmanship Ratio of Grants

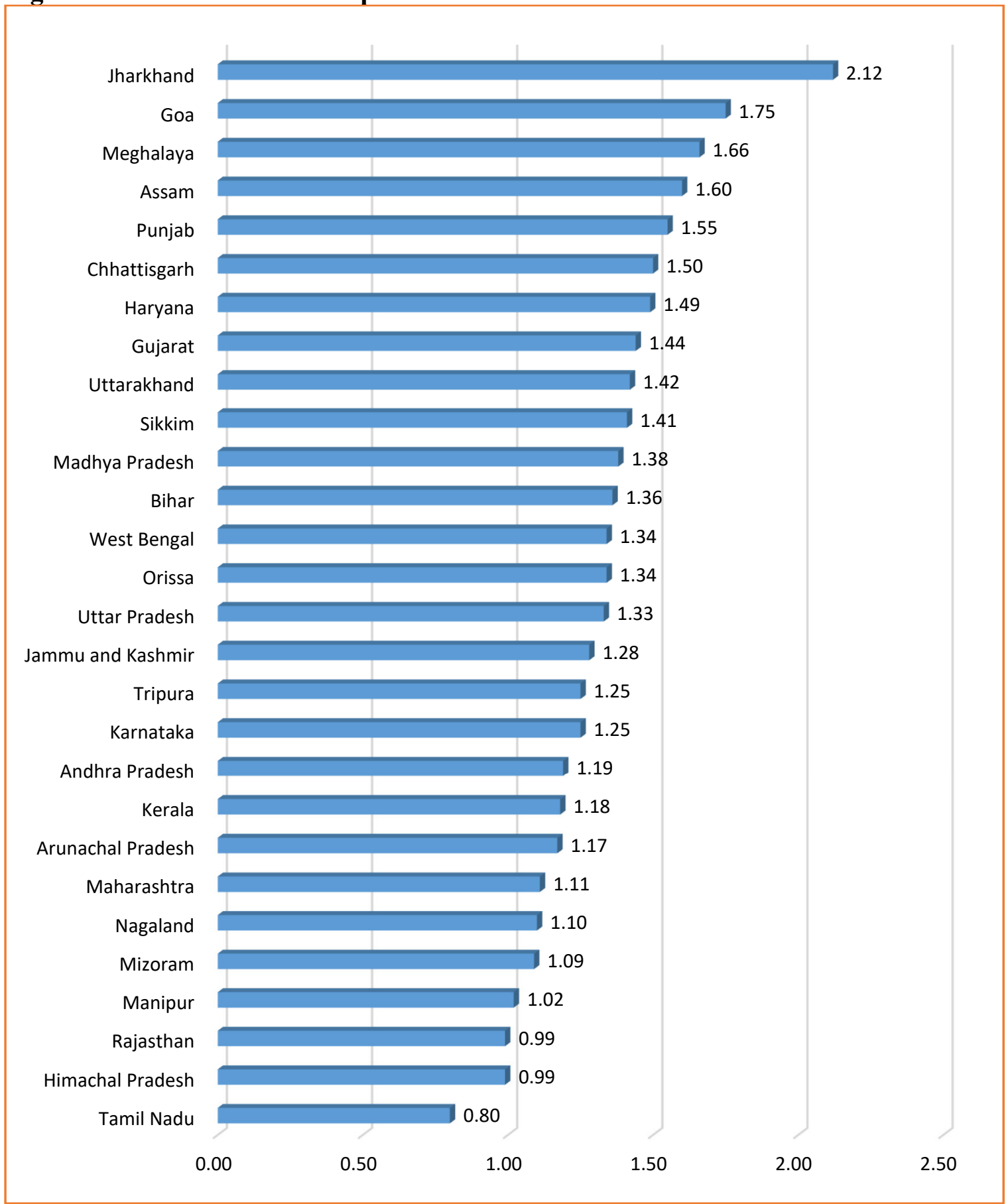

Source: CAG office finance accounts of states and state budget documents (basic data; various years) 
Figure 4: Fiscal Marksmanship Ratio of Revenue Expenditure

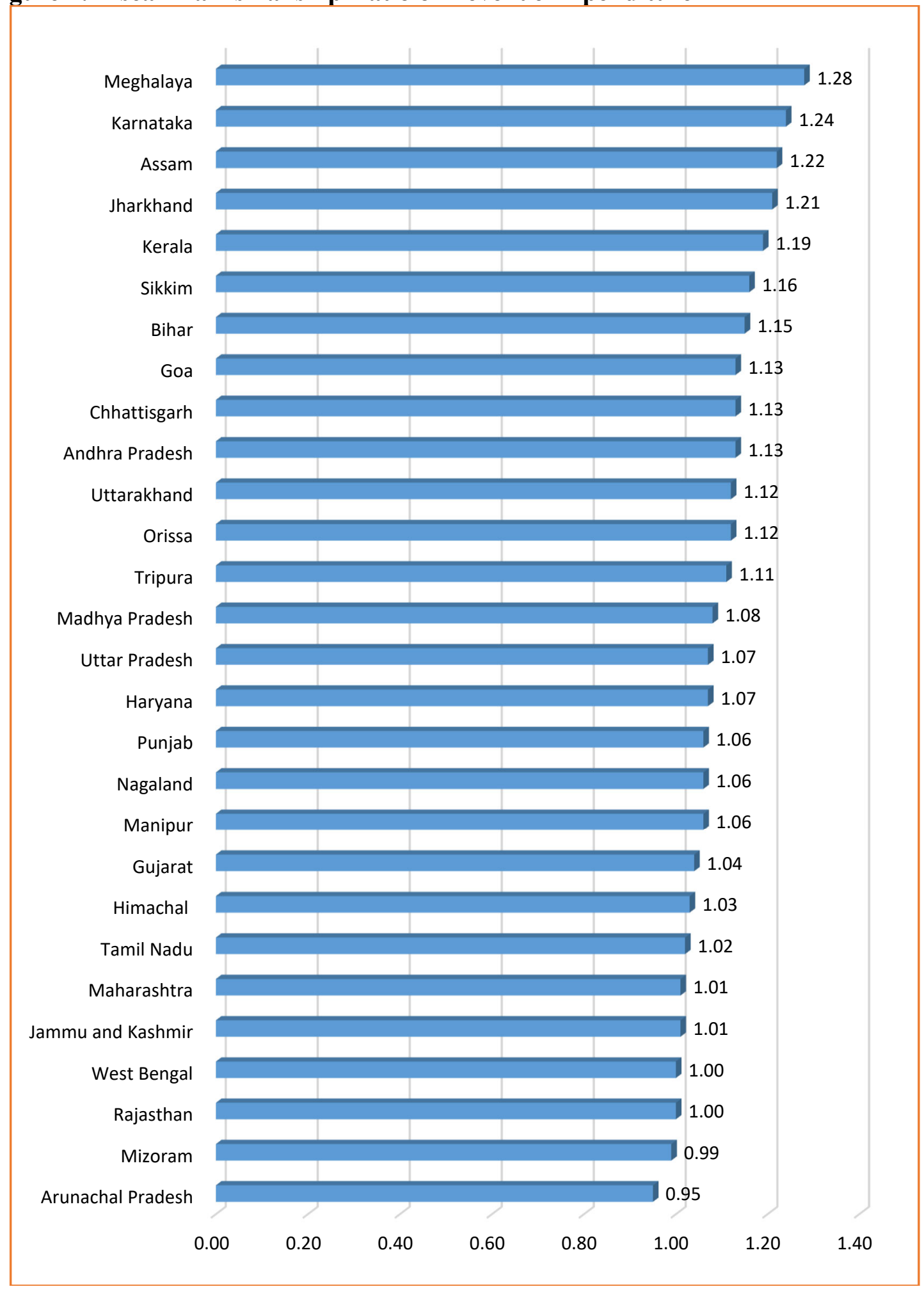

Source: CAG office finance accounts of states and state budget documents (basic data; various years) 
Figure 5: Fiscal Marksmanship Ratio of Capital Expenditure

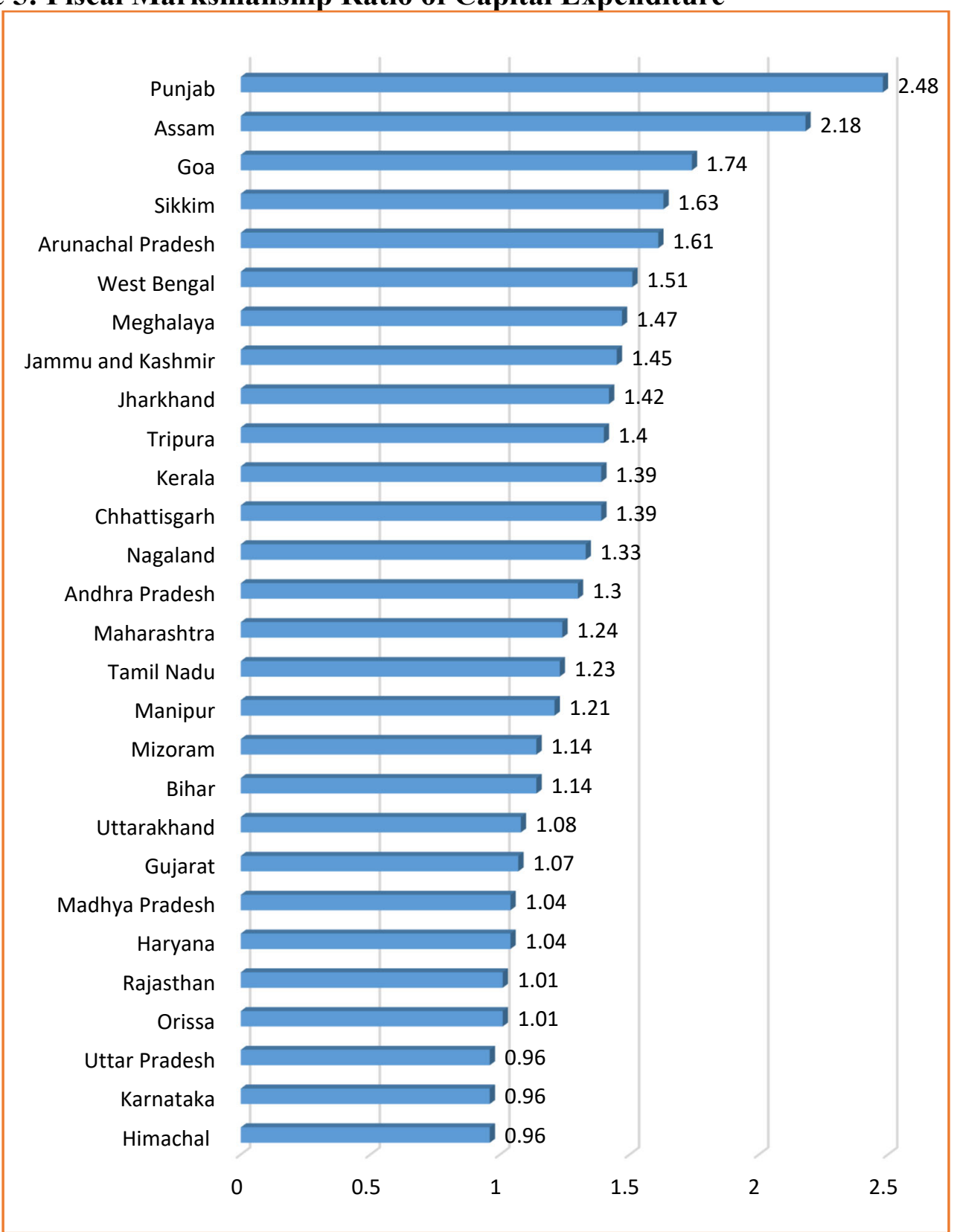

Source: CAG office finance accounts of states and state budget documents (basic data; various years) 


\section{ANALYZING THE FORECAST ERRORS USING THEIL'S U}

The $\mathrm{U}_{1}$ of Theil's index has a lower limit of zero (which is the case of perfect forecast) and an upper limit of one (which is the highest forecasting error). We will state some of the observations on the forecasting errors and elaborate on them using some basic statistical indicators.

In the case of the $\mathrm{BE}$, the average forecasting errors in most of the revenue and expenditure are below 0.2. Furthermore, almost all of the variables (except revenue deficit) are positively skewed (since median $<$ mean). This means that a lot of the observations are clustered to the left side of the interval of $U_{1}$ (i.e., 0 and 1 ) and most of them are below 0.2. One can observe that, on average, both revenue and expenditure variables have low forecasting errors. We observe that the all-state average for total revenue receipt is 0.09 . The all-state average forecast error for the tax revenue is 0.074 and for nontax revenue is 0.15 . In the case of the expenditure variables, the all-India average is 0.08 for revenue expenditure and 0.177 for capital expenditure.

When we look at the error in estimating the fiscal deficit, the $\mathrm{U}_{1}$ for fiscal deficit, on average, is 0.302 . The states that have fiscal deficit forecast errors greater than 0.5 are only four, viz., Arunachal Pradesh (0.818), Assam (0.554), Mizoram (0.617), and Punjab (0.866). In the case of revenue deficit, the value of $U_{1}$ was higher, at 0.432 . Seven states have a $U_{1}$ higher than 0.5, viz., Andhra Pradesh (0.672), Assam (0.94), Goa (0.59), Jammu and Kashmir (0.861), Kerala (0.532), Uttar Pradesh (0.669), and West Bengal (0.636).

A very similar observation can be made regarding the revised estimates (tables 9 and 10). The average forecasting errors for most of the revenue and expenditure variables are below 0.20 . Similar to BE, all the variables are positively skewed. It is worth noting that for most of the major revenue and expenditure variables, $\mathrm{RE}$ is better forecasted than $\mathrm{BE}$ (on average) (tables 9 and 10). For all the macro-fiscal variables among the states, the value of $\mathrm{U}_{1}$ in $\mathrm{RE}$ is lower than $\mathrm{BE}$. 
Applying Theil's U, we have estimated the errors between the BE and the actuals as well as between the RE and actual. As mentioned above, the range of $U_{1}$ is between zero and one, with the value zero for $U_{1}$ equaling a perfect forecast. Figures 6-11 depict the magnitude of errors in the macro-fiscal variables of India's subnational governments. The maximum-minimum range of $\mathrm{U}_{1}$ for $\mathrm{BE}$-actuals revealed that the range of errors in revenue receipts is the higher than that of revenue expenditure and capital expenditure (max of 0.83 in the case of Arunachal Pradesh to a minimum for 0.07 in the case of Uttarakhand; see figure 9). The $\mathrm{U}_{1}$ magnitude of forecasts for the revenue receipts also revealed that around ten states have a magnitude of error greater than 0.30 , viz, Arunachal Pradesh (90.83), Tripura (0.63), Punjab (0.63), Tamil Nadu (0.53), Nagaland (0.53), Mizoram (0.52), Assam (0.51), Jammu and Kashmir (0.47), Goa (0.45), and Uttar Pradesh (0.34).

On the contrary, a magnitude of error above the 0.30 threshold in the case of revenue expenditure is noted for only for Jammu and Kashmir (0.50), Punjab and Assam (0.37), and Arunachal Pradesh (0.30) (figure 10). In the case of capital expenditure, the magnitude of error is highest in Jammu and Kashmir (0.48), followed by Punjab (0.39), Assam (0.37), Goa (0.311), and Arunachal Pradesh (0.25). The minimum error in the capital expenditure forecast is noted for Karnataka at 0.034 (figure 11).

Looking at the end of the tail, around 16 states have a magnitude of error lower than the 0.15 threshold in the case of revenue expenditure (figure 10); on the contrary, the revenue receipts with only eight state having revenue receipt forecast errors less than the 0.15 threshold (figure 9). The lower end of the forecast errors in capital expenditure (below the 0.15 threshold) was noted for 15 states. 
Figure 6: $U_{1}$ for Revenue Deficit: Magnitude of Errors (BE-Actual), 2011-16

U1: Magnitude of Errors in Revenue Deficit Forecasts: BE-Actual

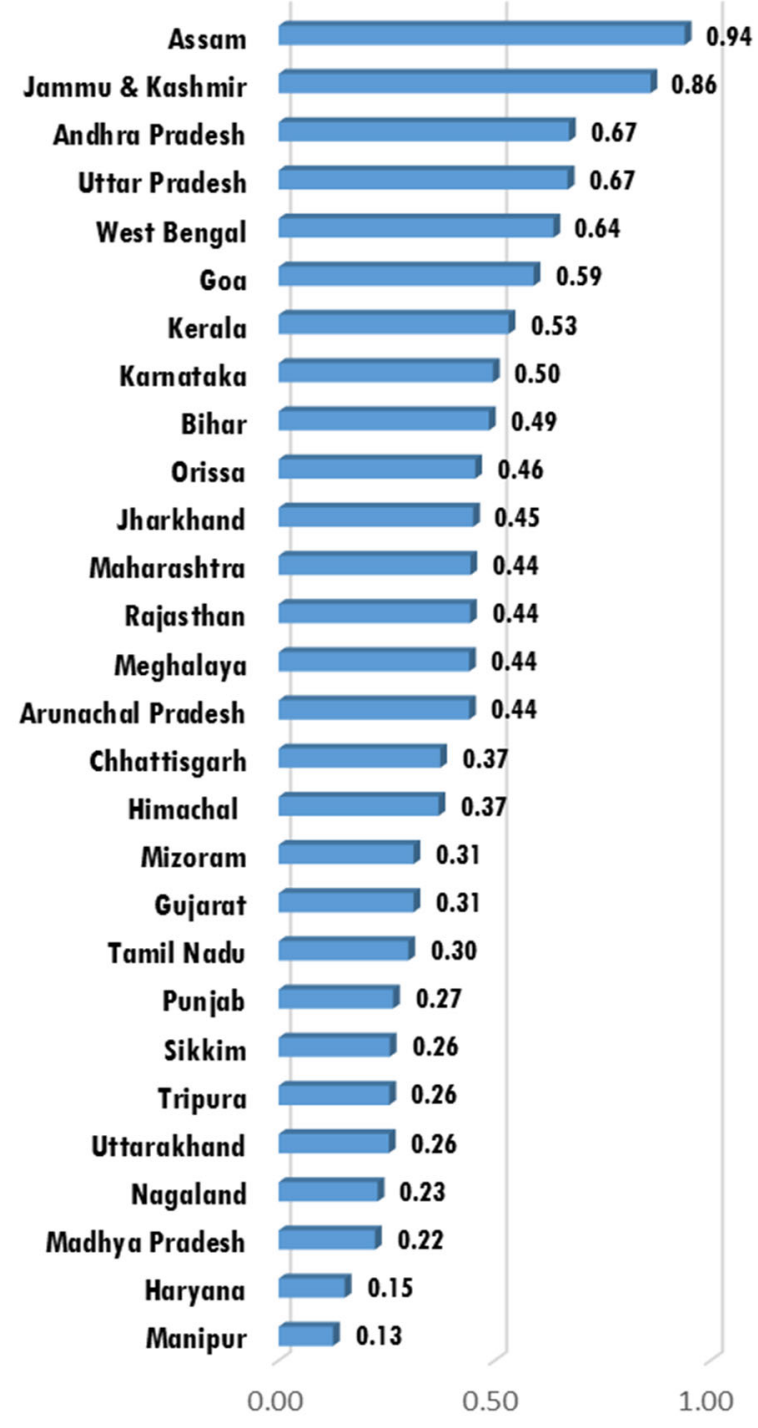

Source: CAG office finance accounts of states and state budget documents (basic data; various years) 
Figure 7: $U_{1}$ for Fiscal Deficit: Magnitude of Errors (BE-Actual), 2011-16

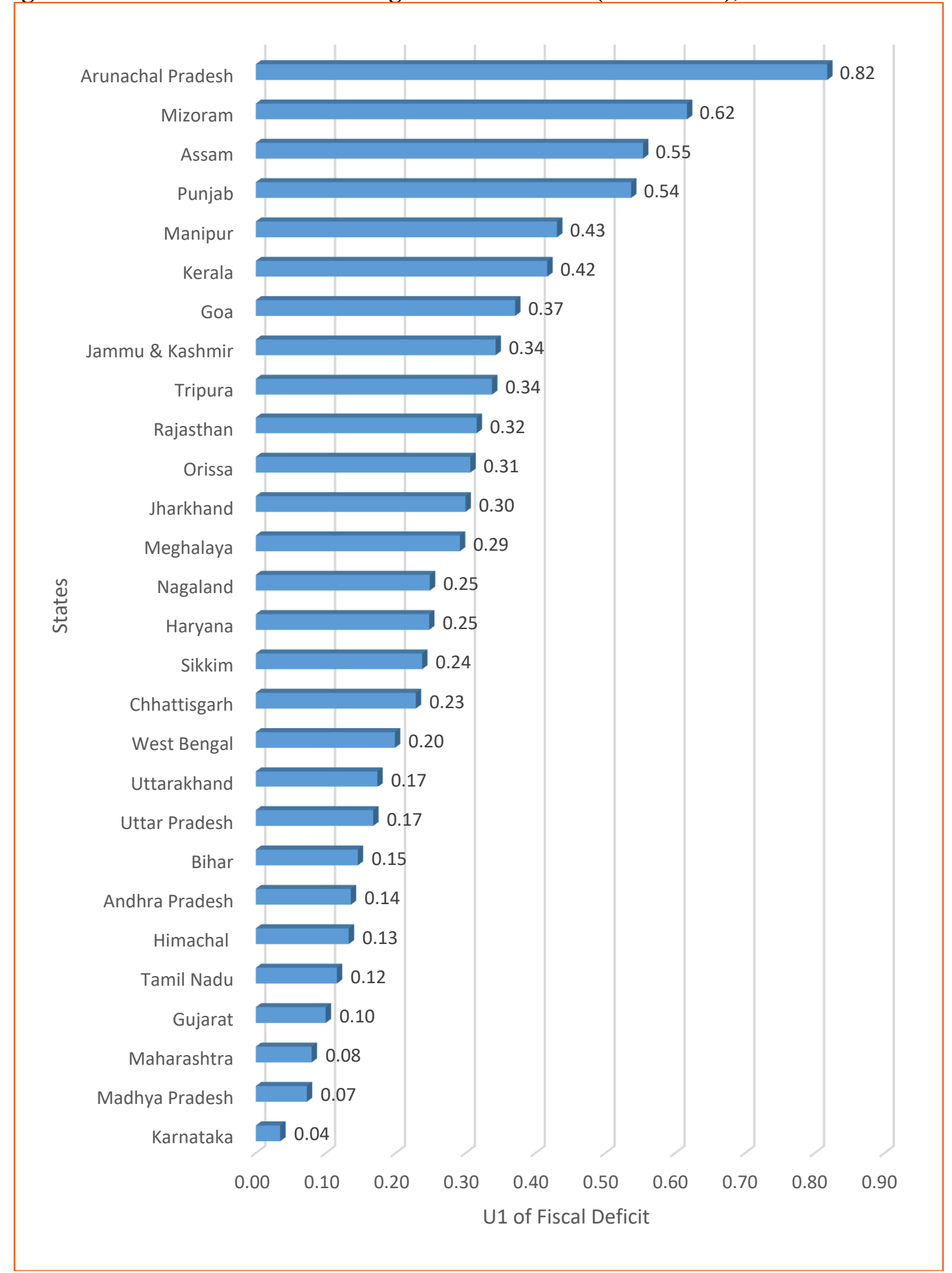

Source: CAG office finance accounts of states and state budget documents (basic data; various years) 
Figure 8: $U_{1}$ for Primary Deficit: Magnitude of Errors (BE-Actual), 2011-16

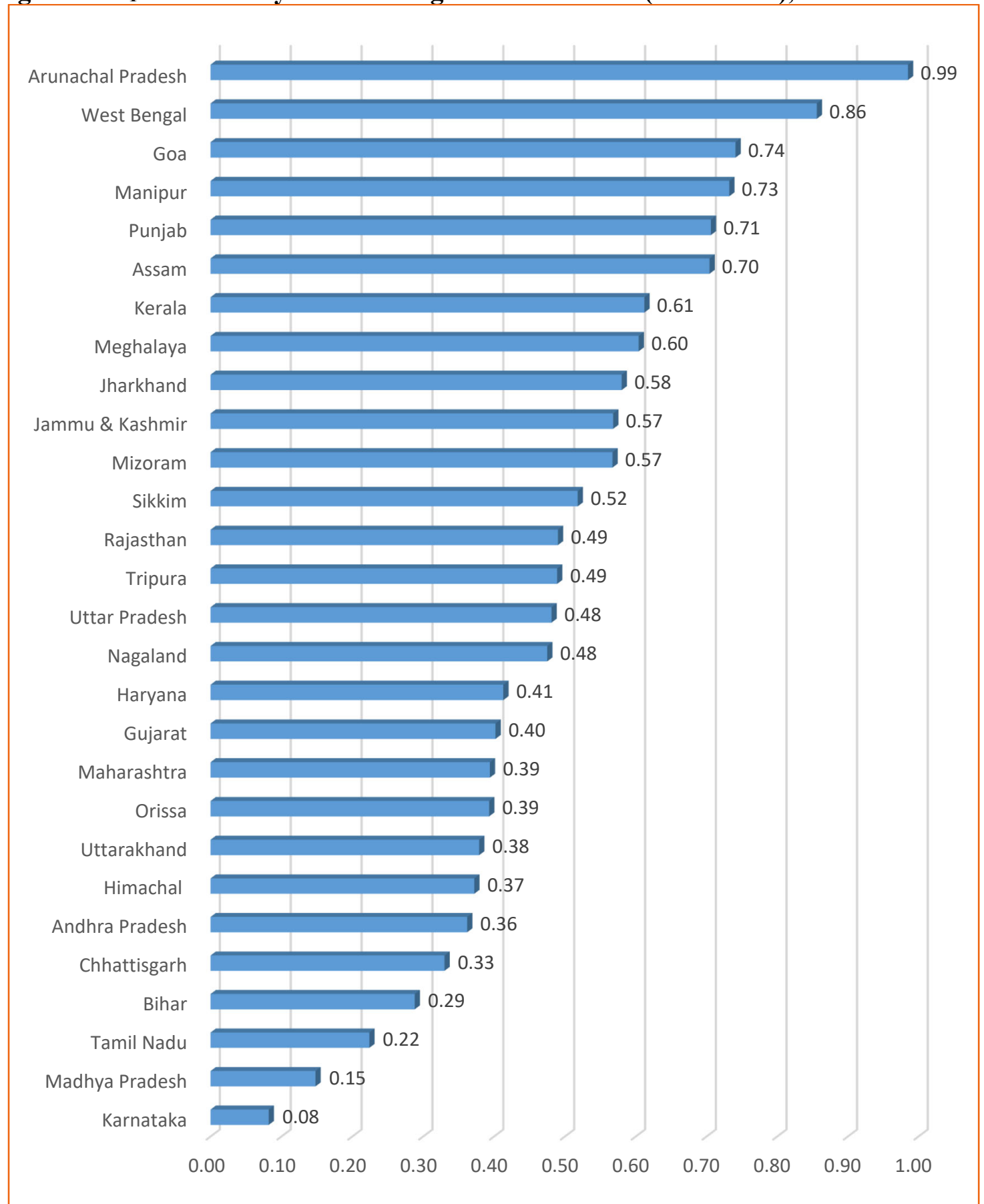

Source: CAG office finance accounts of states and state budget documents (basic data; various years) 
Figure 9: $U_{1}$ for Revenue Receipts: Magnitude of Errors (BE-Actual), 2011-16

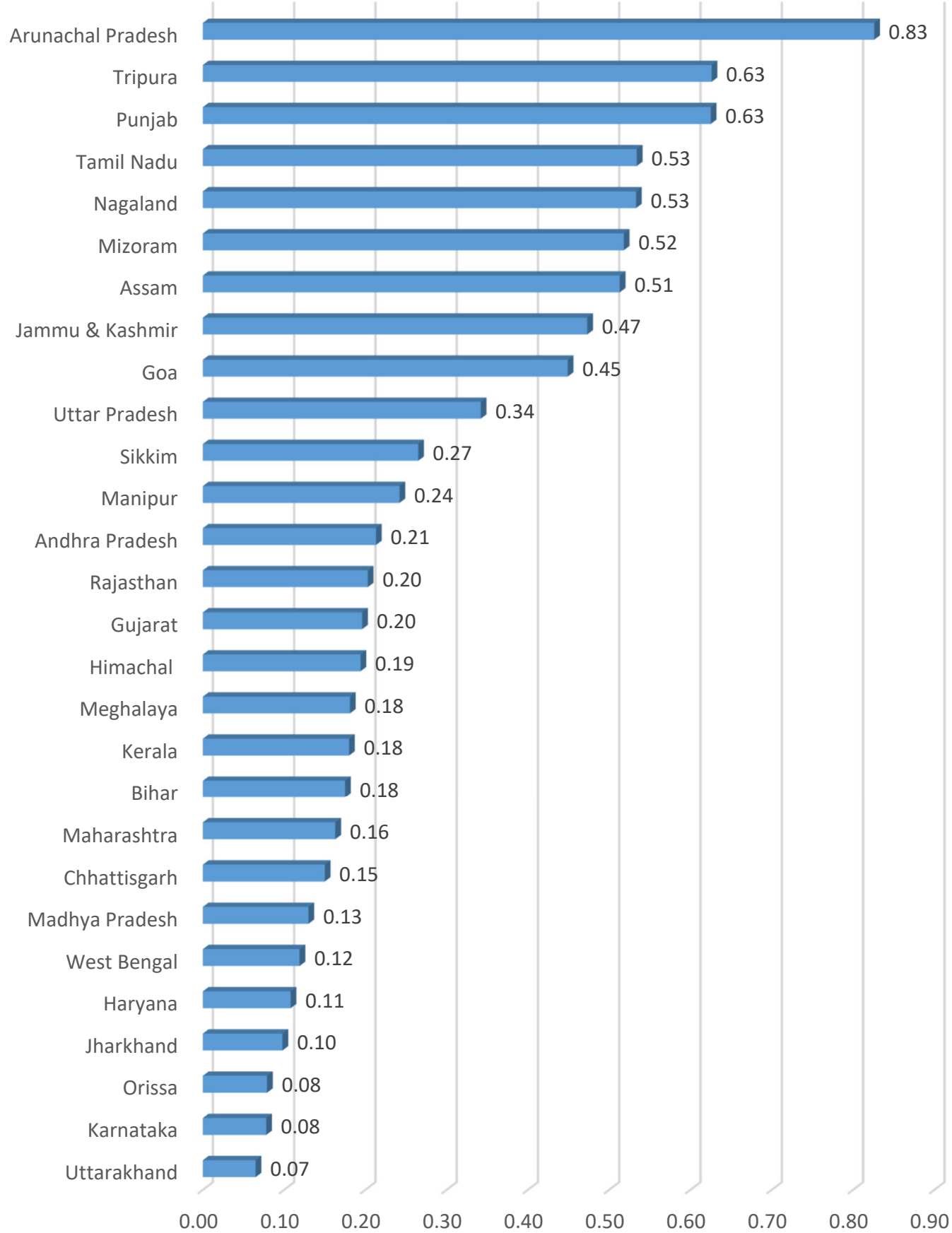

Source: CAG office finance accounts of states and state budget documents (basic data; various years) 
Figure 10: $\mathrm{U}_{1}$ for Revenue Expenditure: Magnitude of Errors (BE-Actual), 2011-16

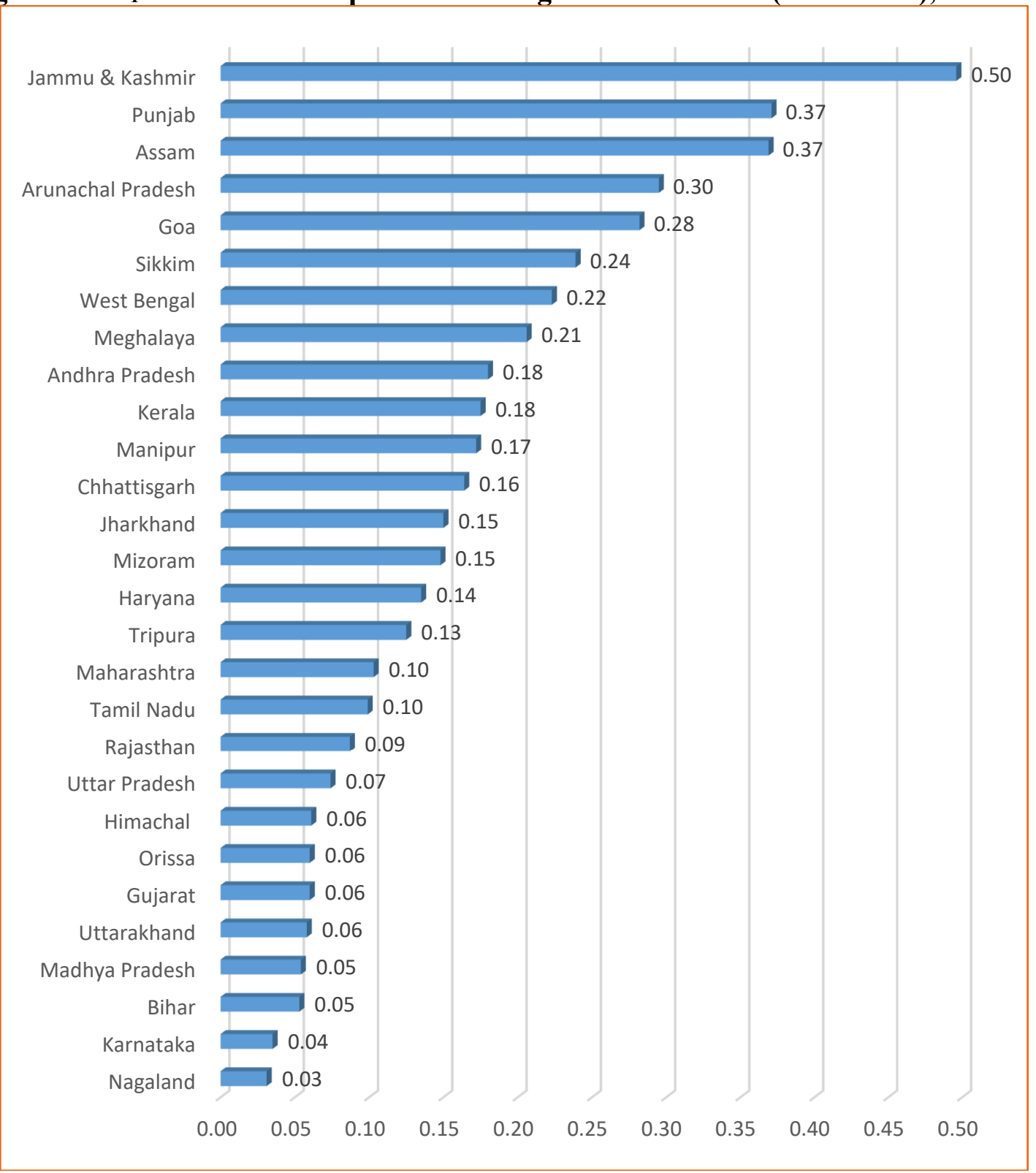

Source: CAG office finance accounts of states and state budget documents (basic data; various years) 
Figure 11: $U_{1}$ for Capital Expenditure: Magnitude of Errors (BE-Actual), 2011-16

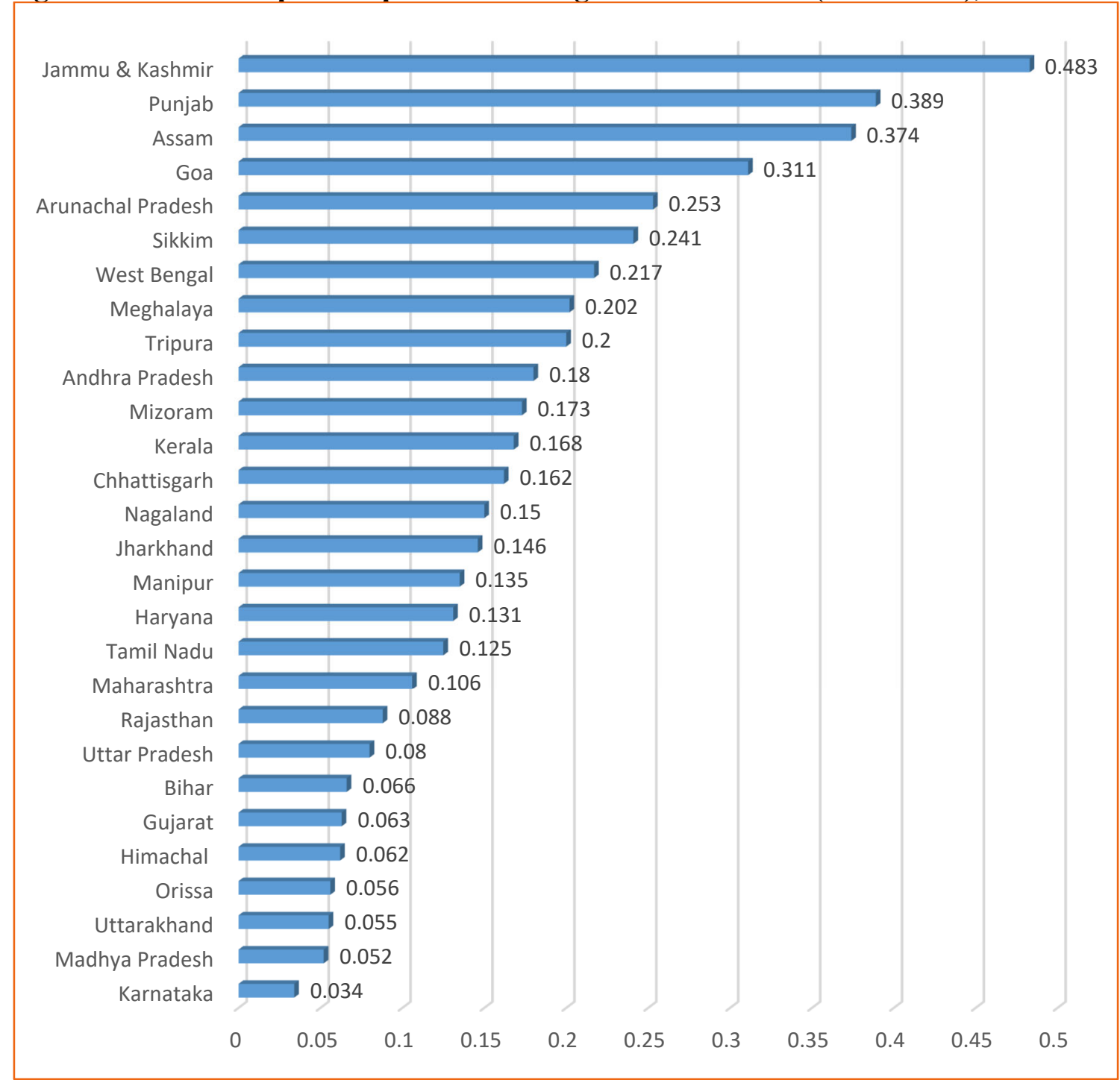

Source: CAG office finance accounts of states and state budget documents (basic data; various years) 
Table 9: Magnitude of Errors in Public Expenditure: Revenue and Capital (comparison of BE-actuals and RE-actuals)

\begin{tabular}{|c|c|c|c|c|}
\hline & \multicolumn{2}{|c|}{ Revenue Expenditure } & \multicolumn{2}{|c|}{ Capital Expenditure } \\
\hline & BE-Actuals & RE-Actuals & BE-Actuals & RE-Actuals \\
\hline Andhra Pradesh & 0.072 & 0.051 & 0.180 & 0.053 \\
\hline Arunachal Pradesh & 0.063 & 0.058 & 0.253 & 0.289 \\
\hline Assam & 0.108 & 0.155 & 0.374 & 0.435 \\
\hline Bihar & 0.077 & 0.119 & 0.066 & 0.127 \\
\hline Chhattisgarh & 0.077 & 0.087 & 0.162 & 0.134 \\
\hline Goa & 0.068 & 0.049 & 0.311 & 0.259 \\
\hline Gujarat & 0.031 & 0.022 & 0.063 & 0.036 \\
\hline Haryana & 0.039 & 0.047 & 0.131 & 0.096 \\
\hline Himachal & 0.027 & 0.041 & 0.062 & 0.042 \\
\hline Jammu and Kashmir & 0.118 & 0.098 & 0.483 & 0.447 \\
\hline Jharkhand & 0.084 & 0.090 & 0.146 & 0.117 \\
\hline Karnataka & 0.168 & 0.170 & 0.034 & 0.042 \\
\hline Kerala & 0.152 & 0.126 & 0.168 & 0.115 \\
\hline Madhya Pradesh & 0.038 & 0.047 & 0.052 & 0.039 \\
\hline Maharashtra & 0.020 & 0.039 & 0.106 & 0.077 \\
\hline Manipur & 0.056 & 0.072 & 0.135 & 0.127 \\
\hline Meghalaya & 0.150 & 0.145 & 0.202 & 0.198 \\
\hline Mizoram & 0.030 & 0.080 & 0.173 & 0.229 \\
\hline Nagaland & 0.056 & 0.072 & 0.150 & 0.129 \\
\hline Orissa & 0.064 & 0.059 & 0.056 & 0.024 \\
\hline Punjab & 0.029 & 0.046 & 0.389 & 0.257 \\
\hline Rajasthan & 0.018 & 0.028 & 0.088 & 0.058 \\
\hline Sikkim & 0.089 & 0.091 & 0.241 & 0.271 \\
\hline Tamil Nadu & 0.026 & 0.024 & 0.125 & 0.074 \\
\hline Tripura & 0.059 & 0.056 & 0.200 & 0.129 \\
\hline Uttarakhand & 0.039 & 0.034 & 0.055 & 0.052 \\
\hline Uttar Pradesh & 0.082 & 0.055 & 0.080 & 0.105 \\
\hline West Bengal & 0.016 & 0.015 & 0.217 & 0.144 \\
\hline
\end{tabular}

Source: CAG office finance accounts of states and state budget documents (basic data; various years) 


\section{Disaggregating the Revenue Receipts}

Some pertinent questions include: Why is it that the forecasting errors are much higher in the revenue receipts in the case of the ten states above the 0.30 threshold as compared to capital expenditure (four states) and revenue expenditure (four states)? Which component of revenue receipts showed erratic range in forecasts - own tax revenue, tax transfers, or grants from the central government?

The disaggregated analysis of revenue receipts showed that the magnitude of errors in grants is relatively higher than the forecast errors in own tax revenue and share in federal taxes. If we take a relative threshold of magnitude of errors at 0.1 , the number of states having forecast errors above 0.1 in the cases of own tax revenue (figure 12) and tax transfers (figure 13) are only three, while the number of states having forecast errors above 0.1 in the case of grants is as high as 23 (figure 14). The three states showing a forecast error magnitude above 0.1 in the case of own tax revenue are Jammu and Kashmir (0.361), Andhra Pradesh (0.157), and Assam (0.101). In the case of tax transfers, the three states that have shown a forecast error magnitude above 0.1 are Jammu and Kashmir (0.361), Tripura (0.17), and Andhra Pradesh (0.114). As many as 23 states have shown forecast errors in grants greater than 0.1, except for Maharashtra (0.088), Nagaland (0.069), Mizoram (0.06), Manipur (0.058), and Himachal Pradesh (0.034) (figure 14). 
Figure 12: Own Tax Revenue: Magnitude of Errors (BE-Actual), 2011-16

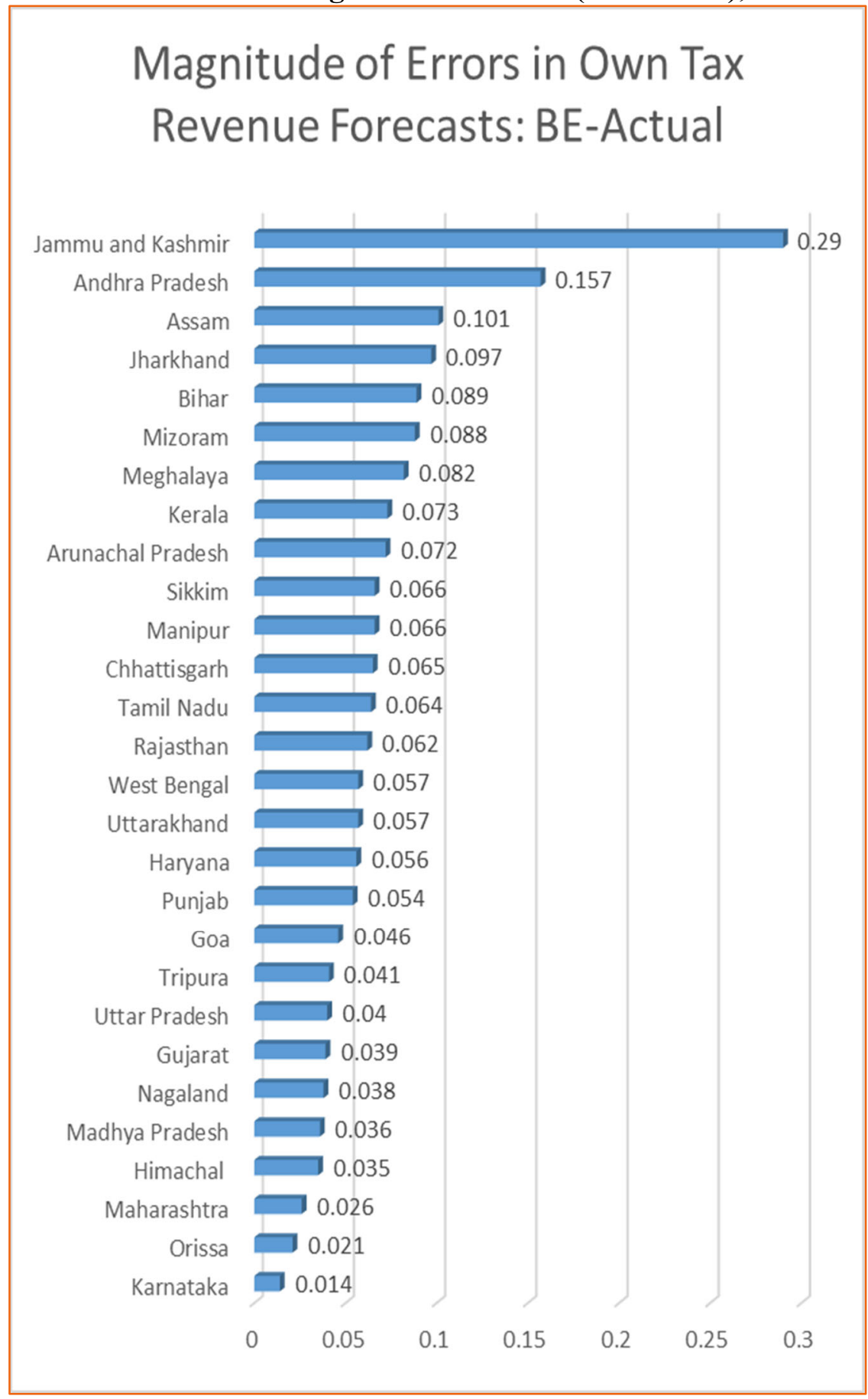

Source: CAG office finance accounts of states and state budget documents (basic data; various years) 
Figure 13: Tax Transfers: Magnitude of Errors (BE-Actual), 2011-16

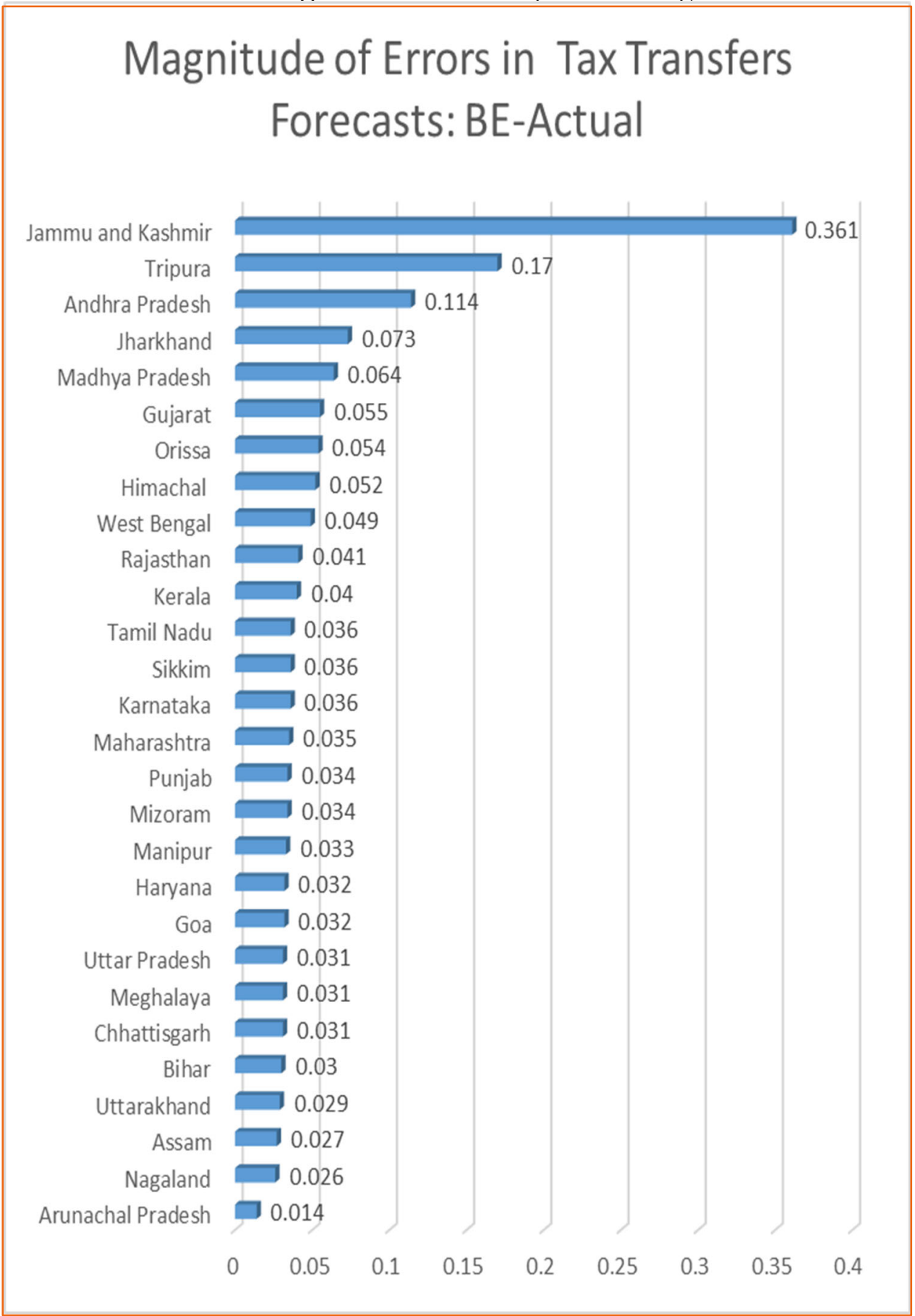

Source: CAG office finance accounts of states and state budget documents (basic data; various years) 
Figure 14: Grants: Magnitude of Errors (BE-Actual), 2011—16

\section{Magnitude of Errors in Grants Forecasts: BE-Actual}

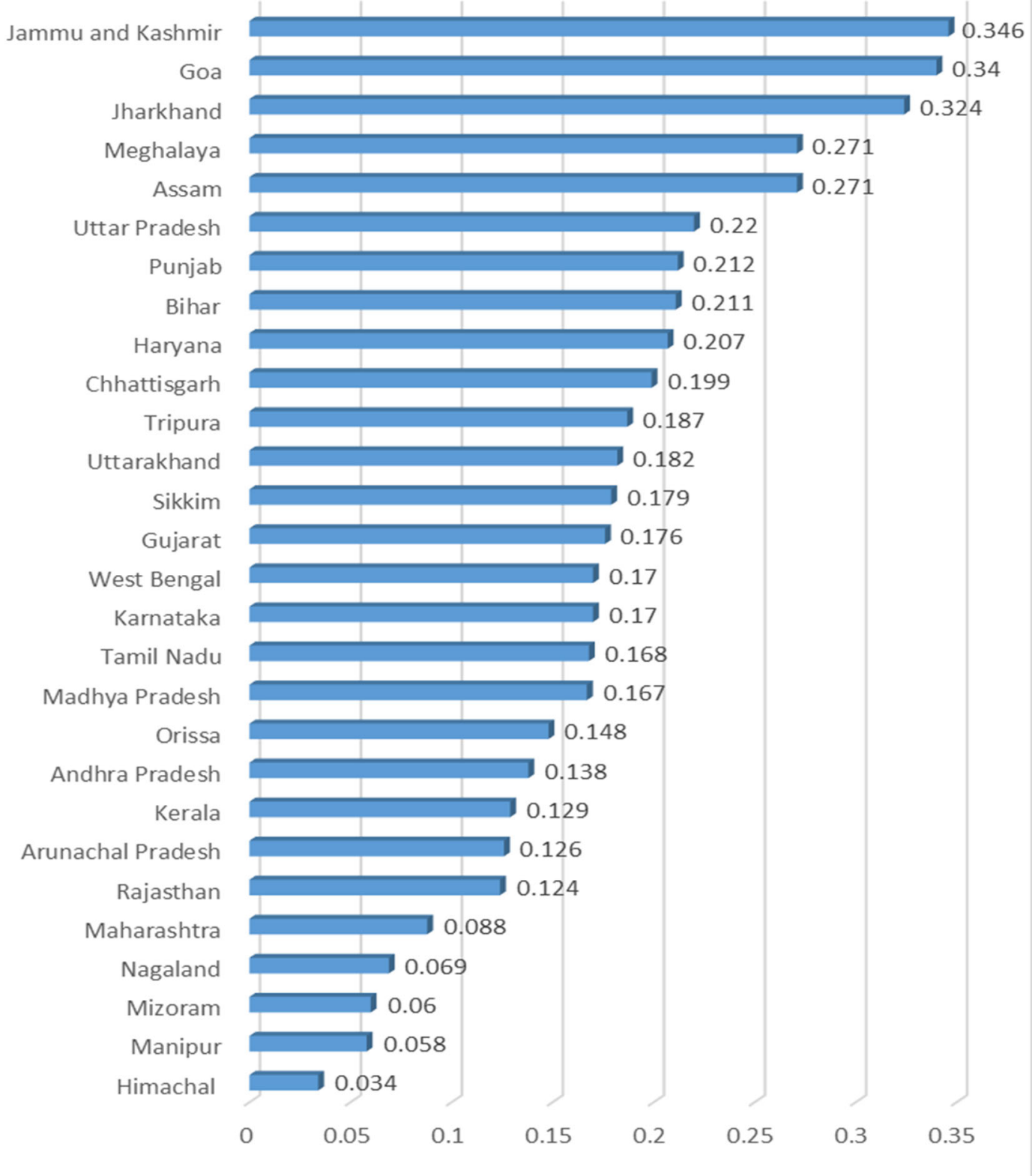

Source: CAG office finance accounts of states and state budget documents (basic data; various years) 
Table 10: Magnitude of Errors: Comparison of BE-Actuals with RE-Actuals for Revenue and its Components

\begin{tabular}{|c|c|c|c|c|c|c|c|c|c|c|}
\hline & \multicolumn{2}{|c|}{ Revenue Receipts } & \multicolumn{2}{|c|}{ Own Tax Revenue } & \multicolumn{2}{|c|}{ Tax Transfers } & \multicolumn{2}{|c|}{ Own Nontax Revenue } & \multicolumn{2}{|c|}{ Grants } \\
\hline & BE-Actuals & RE-Actuals & BE-Actuals & RE-Actuals & BE-Actuals & RE-Actuals & BE-Actuals & RE-Actuals & BE-Actuals & RE-Actuals \\
\hline Andhra Pradesh & 0.121 & 0.043 & 0.157 & 0.018 & 0.114 & 0.002 & 0.099 & 0.018 & 0.138 & 0.162 \\
\hline Arunachal Pradesh & 0.076 & 0.090 & 0.072 & 0.012 & 0.014 & 0.004 & 0.149 & 0.111 & 0.126 & 0.137 \\
\hline Assam & 0.124 & 0.095 & 0.101 & 0.032 & 0.027 & 0.033 & 0.155 & 0.056 & 0.271 & 0.247 \\
\hline Bihar & 0.082 & 0.073 & 0.089 & 0.028 & 0.030 & 0.020 & 0.338 & 0.171 & 0.211 & 0.220 \\
\hline Chhattisgarh & 0.090 & 0.094 & 0.065 & 0.054 & 0.031 & 0.025 & 0.152 & 0.146 & 0.199 & 0.200 \\
\hline Goa & 0.060 & 0.027 & 0.046 & 0.018 & 0.032 & 0.016 & 0.078 & 0.030 & 0.340 & 0.298 \\
\hline Gujarat & 0.043 & 0.047 & 0.039 & 0.072 & 0.055 & 0.026 & 0.067 & 0.046 & 0.176 & 0.147 \\
\hline Haryana & 0.055 & 0.038 & 0.056 & 0.027 & 0.032 & 0.035 & 0.121 & 0.065 & 0.207 & 0.199 \\
\hline Himachal & 0.054 & 0.054 & 0.035 & 0.141 & 0.052 & 0.059 & 0.130 & 0.112 & 0.034 & 0.041 \\
\hline Jammu and Kashmir & 0.271 & 0.266 & 0.290 & 0.315 & 0.361 & 0.353 & 0.188 & 0.237 & 0.346 & 0.317 \\
\hline Jharkhand & 0.120 & 0.116 & 0.097 & 0.205 & 0.073 & 0.021 & 0.133 & 0.184 & 0.324 & 0.277 \\
\hline Karnataka & 0.064 & 0.081 & 0.014 & 0.095 & 0.036 & 0.013 & 0.045 & 0.067 & 0.170 & 0.165 \\
\hline Kerala & 0.054 & 0.063 & 0.073 & 0.085 & 0.040 & 0.017 & 0.065 & 0.028 & 0.129 & 0.119 \\
\hline Madhya Pradesh & 0.161 & 0.145 & 0.036 & 0.221 & 0.064 & 0.024 & 0.122 & 0.048 & 0.167 & 0.135 \\
\hline Maharashtra & 0.051 & 0.040 & 0.026 & 0.069 & 0.035 & 0.001 & 0.161 & 0.078 & 0.088 & 0.181 \\
\hline Manipur & 0.134 & 0.098 & 0.066 & 0.654 & 0.033 & 0.028 & 0.222 & 0.179 & 0.058 & 0.078 \\
\hline Meghalaya & 0.177 & 0.183 & 0.082 & 0.305 & 0.031 & 0.030 & 0.175 & 0.169 & 0.271 & 0.261 \\
\hline Mizoram & 0.095 & 0.115 & 0.088 & 0.532 & 0.034 & 0.023 & 0.116 & 0.103 & 0.060 & 0.089 \\
\hline Nagaland & 0.084 & 0.111 & 0.038 & 0.709 & 0.026 & 0.003 & 0.104 & 0.128 & 0.069 & 0.045 \\
\hline Orissa & 0.088 & 0.117 & 0.021 & 0.221 & 0.054 & 0.016 & 0.121 & 0.075 & 0.148 & 0.158 \\
\hline Punjab & 0.080 & 0.067 & 0.054 & 0.070 & 0.034 & 0.023 & 0.254 & 0.170 & 0.212 & 0.176 \\
\hline Rajasthan & 0.052 & 0.084 & 0.062 & 0.146 & 0.041 & 0.004 & 0.116 & 0.025 & 0.124 & 0.086 \\
\hline Sikkim & 0.066 & 0.061 & 0.066 & 0.547 & 0.036 & 0.026 & 0.145 & 0.121 & 0.179 & 0.245 \\
\hline Tamil Nadu & 0.061 & 0.053 & 0.064 & 0.071 & 0.036 & 0.014 & 0.072 & 0.050 & 0.168 & 0.053 \\
\hline Tripura & 0.091 & 0.071 & 0.041 & 0.255 & 0.170 & 0.027 & 0.130 & 0.067 & 0.187 & 0.078 \\
\hline Uttarakhand & 0.044 & 0.041 & 0.057 & 0.020 & 0.029 & 0.031 & 0.073 & 0.056 & 0.182 & 0.206 \\
\hline Uttar Pradesh & 0.067 & 0.097 & 0.040 & 0.142 & 0.031 & 0.022 & 0.249 & 0.175 & 0.220 & 0.216 \\
\hline West Bengal & 0.122 & 0.141 & 0.057 & 0.223 & 0.049 & 0.025 & 0.270 & 0.200 & 0.170 & 0.116 \\
\hline
\end{tabular}

Source: CAG office finance accounts of states and state budget documents (basic data; various years) 
Table 11: Magnitude of Errors: Comparison of BE-Actuals with RE-Actuals for Revenue Deficit, Fiscal Deficit, and Primary Deficit

\begin{tabular}{|c|c|c|c|c|c|c|}
\hline & \multicolumn{2}{|c|}{ Revenue Deficit } & \multicolumn{2}{|c|}{ Fiscal Deficit } & \multicolumn{2}{|c|}{ Primary Deficit } \\
\hline & BE-Actuals & RE-Actuals & BE-Actuals & RE-Actuals & BE-Actuals & RE-Actuals \\
\hline Andhra Pradesh & 0.672 & 0.640 & 0.136 & 0.073 & 0.362 & 0.240 \\
\hline Arunachal Pradesh & 0.441 & 0.441 & 0.818 & 0.643 & 0.985 & 0.718 \\
\hline Assam & 0.940 & 0.462 & 0.554 & 0.293 & 0.704 & 0.341 \\
\hline Bihar & 0.487 & 0.589 & 0.146 & 0.347 & 0.288 & 0.499 \\
\hline Chhattisgarh & 0.374 & 0.336 & 0.229 & 0.248 & 0.330 & 0.359 \\
\hline Goa & 0.590 & 0.551 & 0.371 & 0.364 & 0.741 & 0.736 \\
\hline Gujarat & 0.312 & 0.229 & 0.100 & 0.056 & 0.402 & 0.221 \\
\hline Haryana & 0.153 & 0.116 & 0.248 & 0.150 & 0.413 & 0.220 \\
\hline Himachal & 0.370 & 0.428 & 0.133 & 0.054 & 0.372 & 0.376 \\
\hline $\begin{array}{l}\text { Jammu and } \\
\text { Kashmir }\end{array}$ & 0.861 & 0.885 & 0.343 & 0.387 & 0.568 & 0.653 \\
\hline Jharkhand & 0.450 & 0.413 & 0.300 & 0.163 & 0.580 & 0.273 \\
\hline Karnataka & 0.496 & 0.302 & 0.035 & 0.051 & 0.082 & 0.103 \\
\hline Kerala & 0.532 & 0.501 & 0.417 & 0.376 & 0.612 & 0.572 \\
\hline Madhya Pradesh & 0.223 & 0.204 & 0.073 & 0.108 & 0.148 & 0.173 \\
\hline Maharashtra & 0.444 & 0.603 & 0.080 & 0.116 & 0.394 & 0.390 \\
\hline Manipur & 0.126 & 0.115 & 0.431 & 0.329 & 0.732 & 0.477 \\
\hline Meghalaya & 0.441 & 0.434 & 0.292 & 0.283 & 0.604 & 0.595 \\
\hline Mizoram & 0.312 & 0.438 & 0.617 & 0.446 & 0.567 & 0.513 \\
\hline Nagaland & 0.229 & 0.332 & 0.249 & 0.430 & 0.475 & 0.812 \\
\hline Orissa & 0.455 & 0.251 & 0.307 & 0.236 & 0.393 & 0.269 \\
\hline Punjab & 0.265 & 0.148 & 0.537 & 0.189 & 0.706 & 0.251 \\
\hline Rajasthan & 0.443 & 0.123 & 0.316 & 0.055 & 0.490 & 0.075 \\
\hline Sikkim & 0.257 & 0.256 & 0.238 & 0.260 & 0.518 & 0.516 \\
\hline Tamil Nadu & 0.300 & 0.138 & 0.116 & 0.039 & 0.224 & 0.068 \\
\hline Tripura & 0.256 & 0.125 & 0.338 & 0.307 & 0.489 & 0.482 \\
\hline Uttarakhand & 0.255 & 0.154 & 0.174 & 0.051 & 0.379 & 0.104 \\
\hline Uttar Pradesh & 0.669 & 0.644 & 0.168 & 0.155 & 0.481 & 0.387 \\
\hline West Bengal & 0.636 & 0.166 & 0.199 & 0.067 & 0.856 & 0.399 \\
\hline
\end{tabular}

Source: CAG office finance accounts of states and state budget documents (basic data; various years) 


\section{Decomposition of Forecast Errors}

We have decomposed the error between the systematic and unsystematic errors. Systematic error is the sum of the ME and slope error. The systematic error can be improved by using better forecasting techniques. The partitioning of sources of state-specific forecast errors is given in appendix 1. Within $\mathrm{BE}$-actuals partitioning, more than 20 states showed that the source of errors was systemic for capital expenditure.

Figure 15: Randomness of Errors in Revenue Deficit (BE-Actual), 2011-16

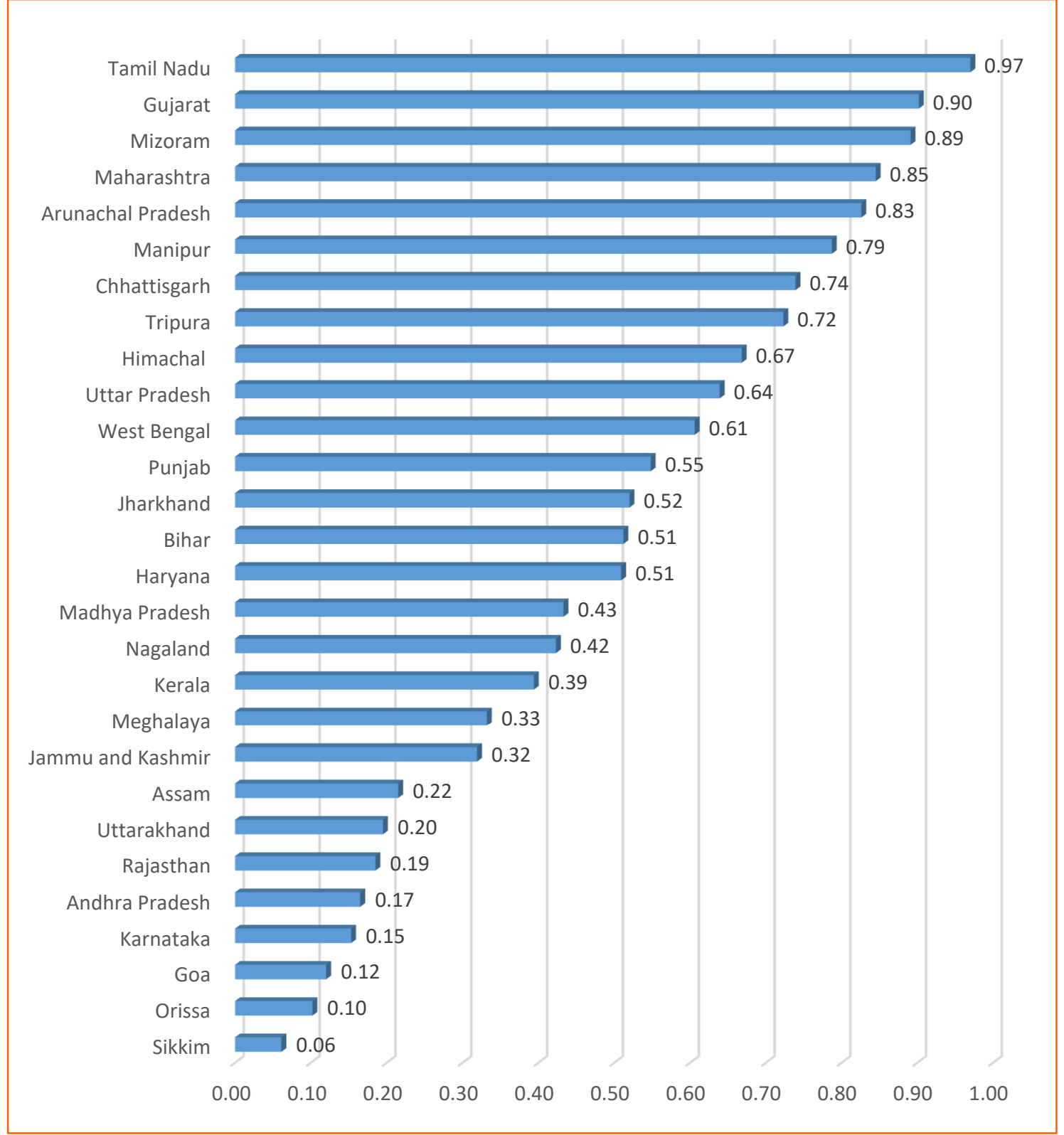

Source: CAG office finance accounts of states and state budget documents (basic data; various years) 
One can observe that in merely seven and five states in the BE and RE, respectively, the capital expenditure variables have the random error of more than 0.5 . The average of the random errors of the $\mathrm{BE}$ and $\mathrm{RE}$ is 0.31 and 0.24 , respectively. Both the above observations tell us that the errors in capital expenditure are more because of systematic bias rather than being random. 
Figure 16: Randomness of Errors in Fiscal Deficit (BE-Actual), 2011-16

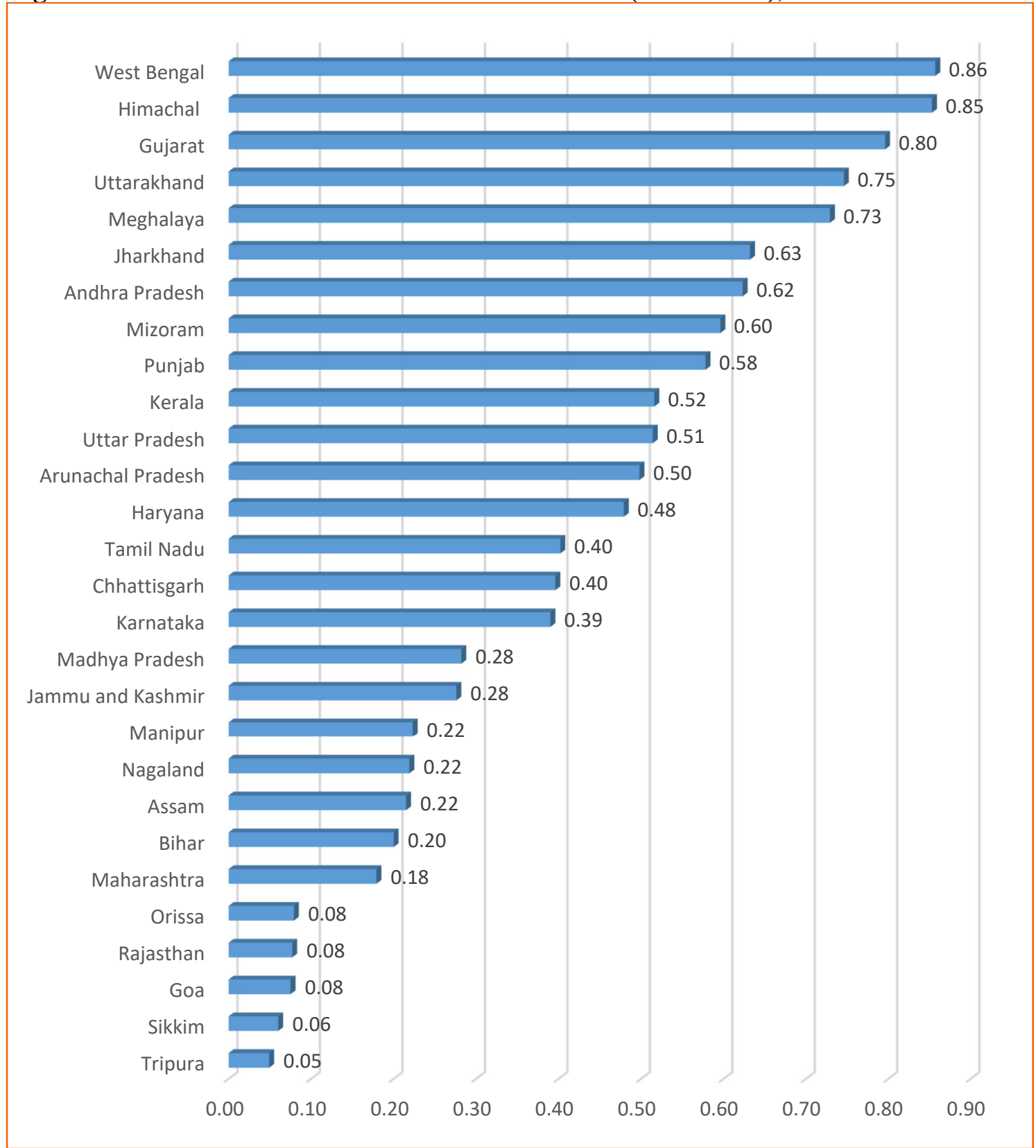

Source: CAG office finance accounts of states and state budget documents (basic data; various years) 
Figure 17: Randomness of Errors in Primary Deficit (BE-Actual), 2011-16

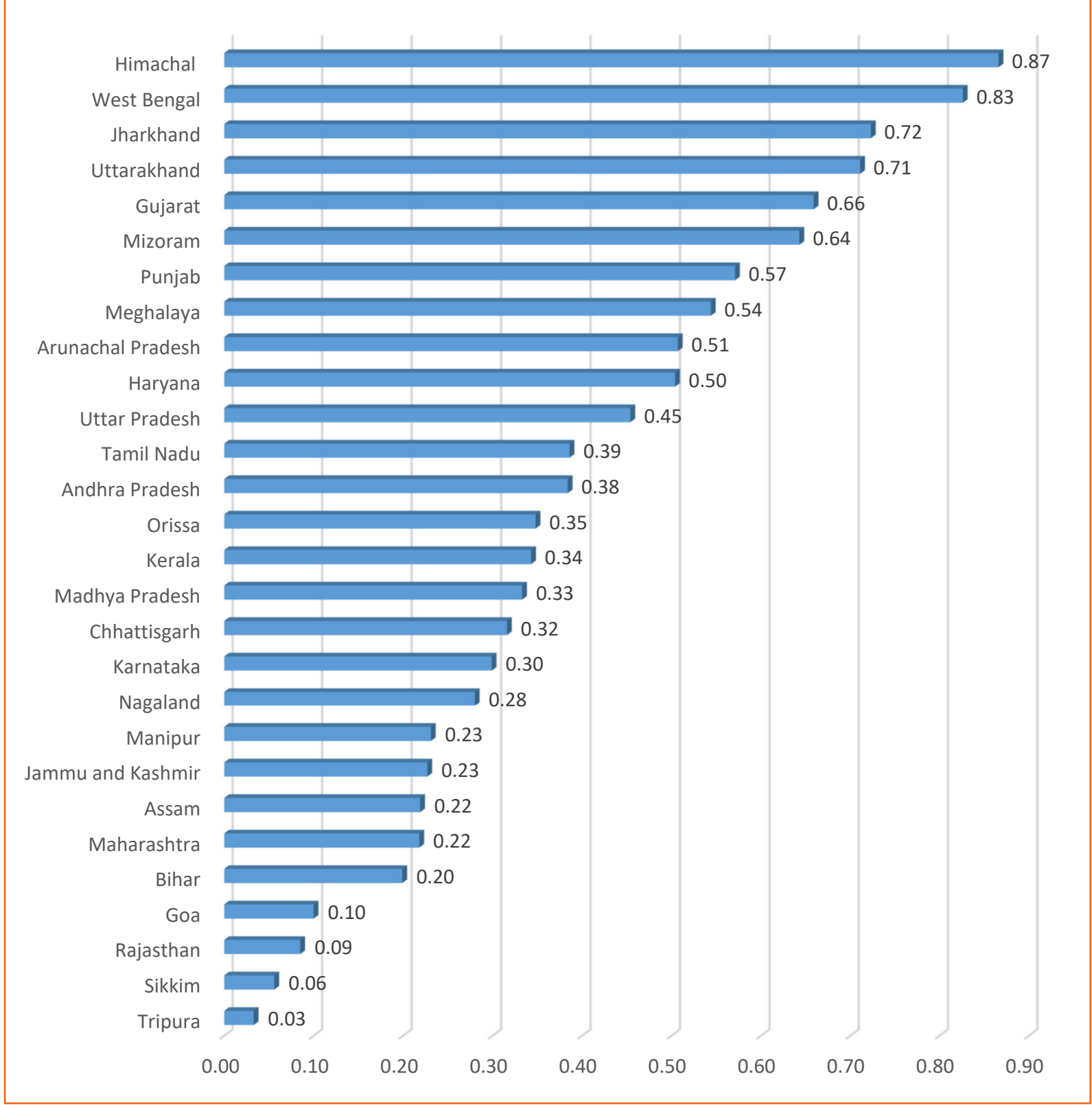

Source: CAG office finance accounts of states and state budget documents (basic data; various years) 
Figure 18: Randomness of Errors in Own Tax Revenue (BE-Actual), 2011-16

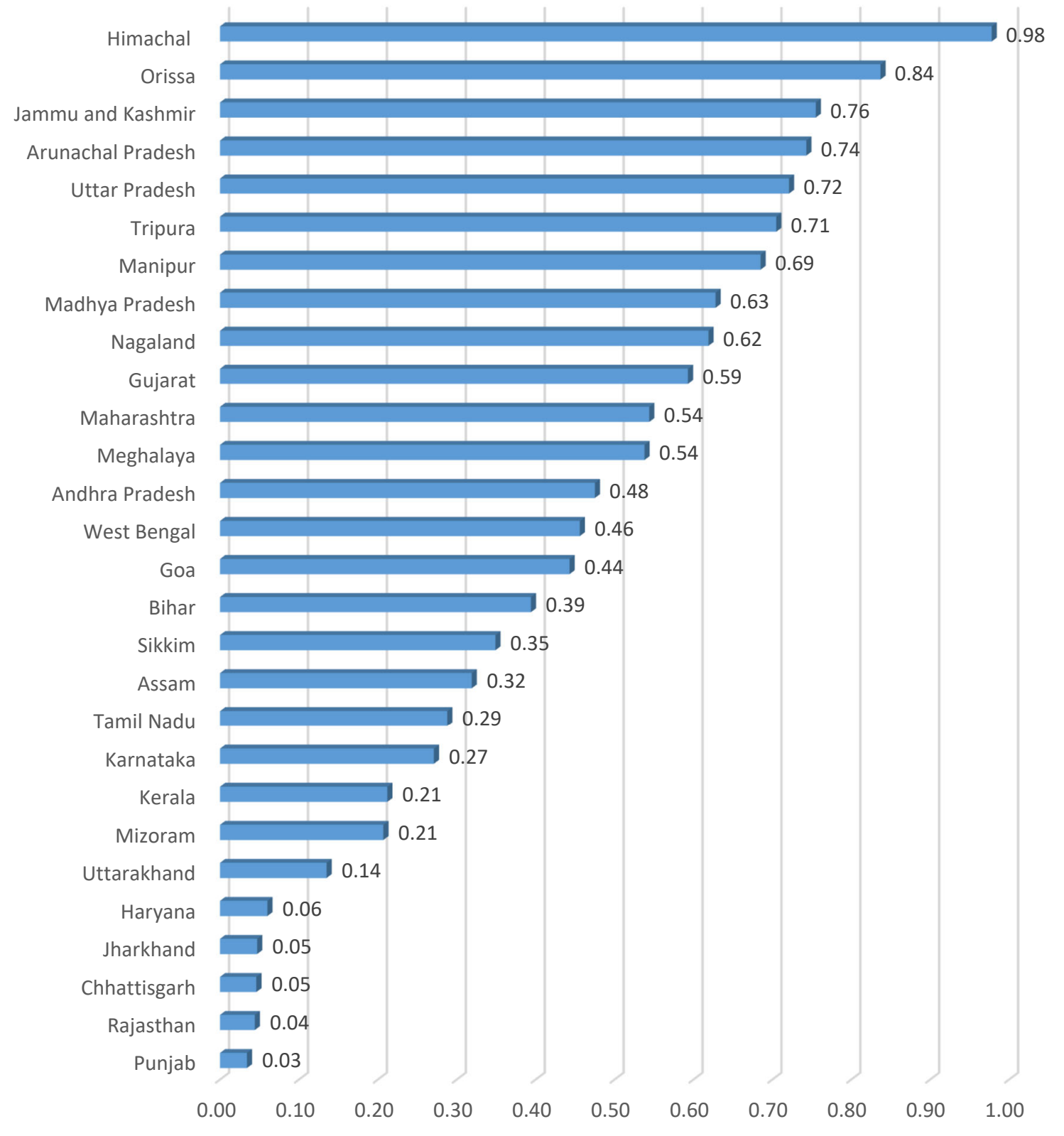

Source: CAG office finance accounts of states and state budget documents (basic data; various years) 
Figure 19: Randomness of Errors in Tax Transfers (BE-Actual), 2011-16

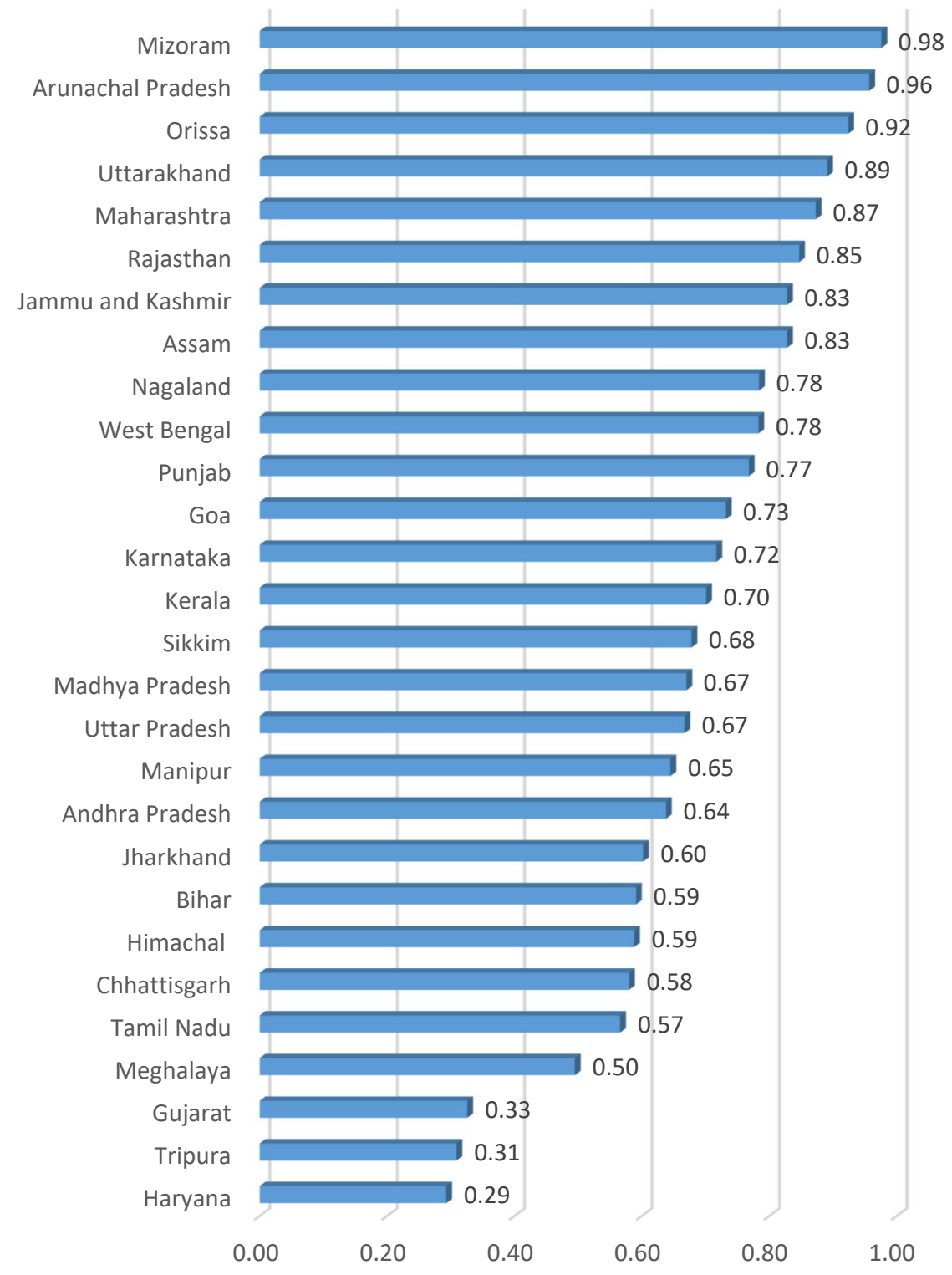

Source: CAG office finance accounts of states and state budget documents (basic data; various years) 
Figure 20: Randomness of Errors in Revenue Expenditure (BE-Actual), 2011-16

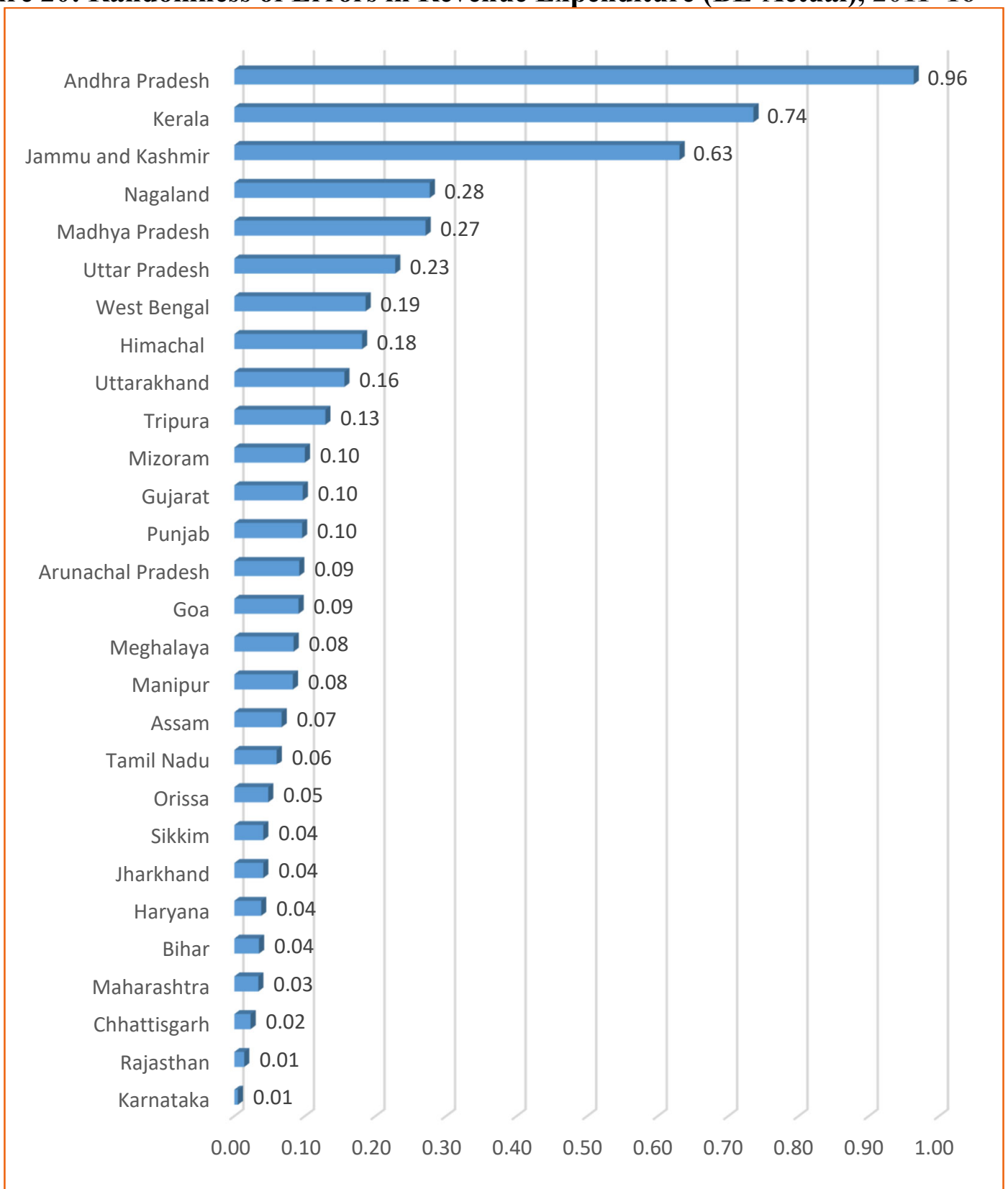

Source: CAG office finance accounts of states and state budget documents (basic data; various years) 
Figure 21: Randomness of Errors in Capital Expenditure (BE-Actual), 2011-16

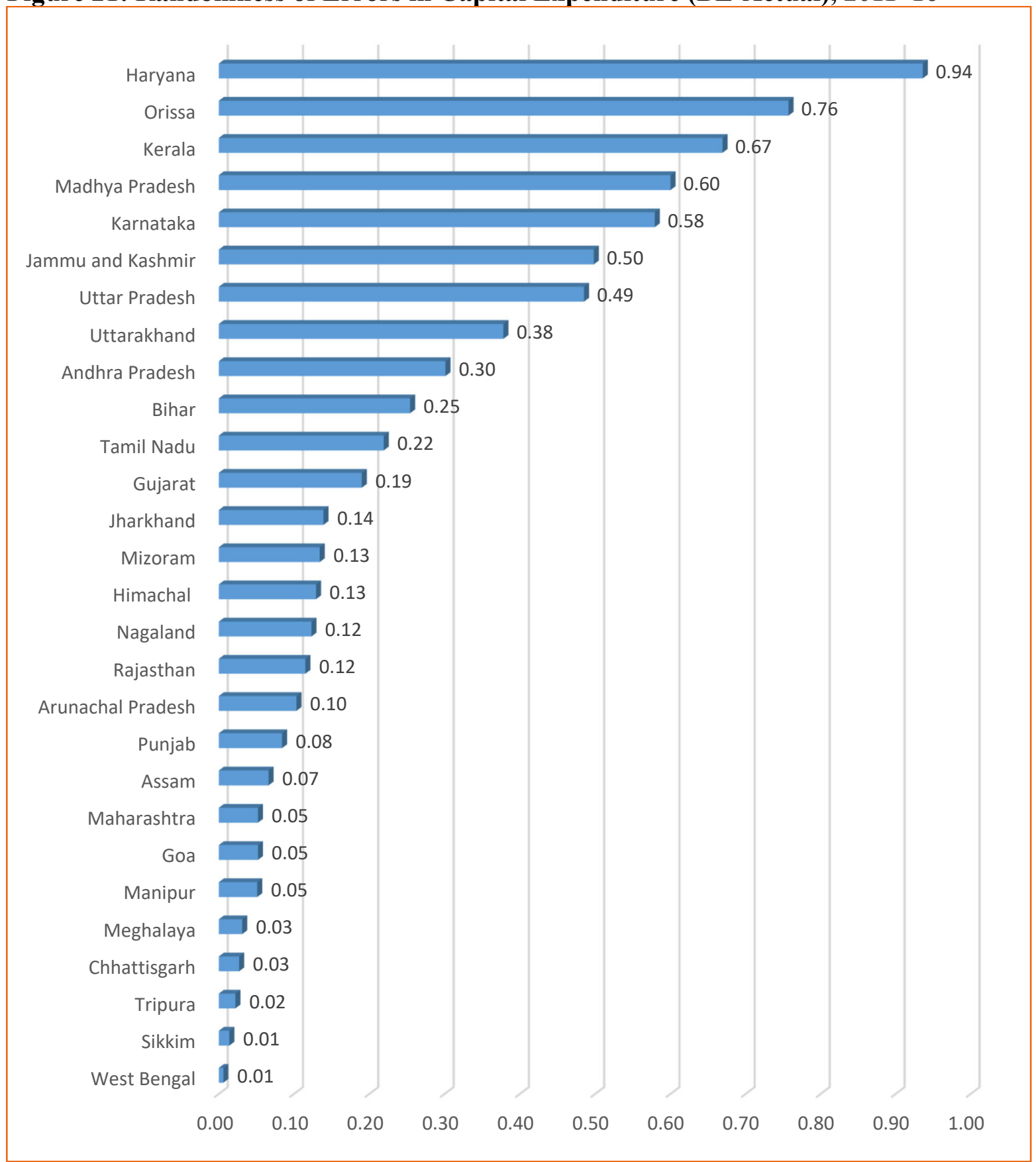

Source: CAG office finance accounts of states and state budget documents (basic data; various years) 
In the case of BE, it can be observed that in most of the categories more than half of the states have a random error of over 50 percent of the total error. In the appendix tables on the sources of errors, it is observed that in the case of all of the revenue and expenditure variables (except share in federal taxes, where four states have a systematic error of over 50 percent), the former trend of random error over 50 percent persists.

While there have been negligible improvements from the BE to the RE- that is, on average, the number of states having a systematic error of more than 50 percent has changed marginally across the different categories of revenues and expenditure - the larger trend of the BE seems to persist. For instance, in categories such as tax revenue, state's own tax revenue, share in federal taxes, state's own non tax revenue, revenue expenditure, revenue deficit, and primary deficit, the number of states having systematic error of over 50 percent of the total error has decreased. For the rest of the categories, the same categories have increased. However, in both cases, the change has been only marginal.

\section{CONCLUSION}

Applying Theil's U technique, we tried to analyze the errors in the fiscal forecasts of India's subnational governments. The fiscal marksmanship analysis showed that the forecast errors in revenue receipts have been greater than for revenue expenditure. Within revenue receipts, the forecast errors in grants is the highest. Within public expenditure, the errors of capital expenditure forecasts showed greater deviations than revenue expenditure. The analysis shows that in more than 20 states, the sources of error are systemic rather than random in the case of a few macro-fiscal variables, with negligible improvements from BE to RE. 


\section{REFERENCES}

Alesina, A., and R. Perotti. 1995. "The Political Economy of Budget Deficits." IMF Staff Papers 42(1).

Allan, C. M. 1965. “Fiscal Marksmanship, 1951-63.” Oxford Economic Papers (New Series) 17(2): 317-27.

Artis, M. J., and M. Marcellino. 2001. "Fiscal Forecasting: The Track Record of the IMF, OECD and EC." Econometrics Journal 4(1): S20-S36.

Asher, M. G. 1978. "Accuracy of Budgetary Forecasts of central Government, 1967-68 to 1975-76." Economic and Political Weekly 13(8).

Auerbach, A. J. 2017. "Fiscal Policy." Paper presented in the Conference on "Rethinking Macroeconomics." Peterson Institute of International Economics, Washington DC, October 12-13.

Auld, D. A. L. 1970. "Fiscal Marksmanship in Canada." The Canadian Journal of Economics 3(3): 507-11.

Beetsma, R. M. W. J., and H. Jensen. 2004. "Mark-Up Fluctuations and Fiscal Policy Stabilization in a Monetary Union." Journal of Macroeconomics 26: 357-76.

Buiter, W. H., and U. R. Patel. 2011. "Fiscal Rules in India: Are they effective?" CEPR Discussion Paper Series No. 7810. London: Centre for Economic Policy Research.

Blanchard, O. 1990. "Suggestions for a New Set of Fiscal Indicators." OECD Working Paper No. 79. Paris: Organisation for Economic Co-operation and Development.

Bretschneider, S. I., W. L. Gorr, G. Grizzle, and E. Klay. 1989. "Political and Organizational influences on the Accuracy of Forecasting State Government Revenues." International Journal of Forecasting 5: 307-19.

Brück, T., and A. Stephan. 2005. "Do Eurozone Countries Cheat with their Budget Deficit Forecasts?” Europa-Universität Viadrina Frankfurt (Oder) Postgraduate Research Programme Working Paper No. 2005.5. Frankfurt: European University Viadrina Frankfurt (Oder).

Cabanillas, L. G., and A. Terzi. 2012. "The accuracy of the European Commission's forecasts re-examined." EC European Papers No. 476. Brussels: European Commission.

Cassidy, G., M. S. Kamlet, and D. S. Nagin. 1989. "An Empirical Examination of Bias in Revenue Forecasts by State Governments." International Journal of Forecasting 5(3): $321-31$. 
Chakraborty, L., and D. Sinha. 2018. "Has Fiscal Rule Changed the Fiscal Marksmanship of Union Government.” NIPFP Working Paper No. 234. New Delhi: National Institute of Public Finance and Policy (NIPFP).

Chakraborty, L., and P. Chakraborty. 2018. "New FRBM Framework: Time to Recast Union Government Expenditure Needs." Economic and Political Weekly 53(9).

Chakrabarty, T. K., and W. Varghese. 1982. "The Government of India's Budget Estimation: An Analysis of the Error Components." Reserve Bank of India, Occasional Papers 3(2): 175-90.

Davis, J. M. 1980. "Fiscal Marksmanship in the United Kingdom, 1951-1978." The Manchester School 48(2): 111-28.

Giuriato, L., A. Cepparulo, and M. Barberi. 2016. "Fiscal forecasts and political systems: a legislative budgeting perspective." Public Choice 168(1): 1-22.

Intriligator, M. D. 1978. Econometric Models, Techniques, and Applications. Upper Saddle River, NJ: Prentice-Hall.

Kopits, G. 2001. "Fiscal Rules: Useful Policy Framework or Unnecessary Ornament?" IMF Working Paper No. 01/145. Washington, DC: International Monetary Fund.

Kumari, A., and B. B. Bhattacharya. 1988. "Budget Forecasts of Central Government Revenue and Expenditure: A Test of Rational Expectation." Economic and Political Weekly 23(26).

Ministry of Finance. 2017. "Fiscal Responsibility and Budget Management (FRBM) Committee Report." New Delhi: Government of India, Ministry of Finance.

Morrison, R. J. 1986. "Fiscal Marksmanship in the United States: 1950-1983." The Manchester School 54(3): 322-33.

Muth, J. F. 1961. "Rational Expectations and the Theory of Price Movements." Econometrica 29: 315-35.

Nitan, K., and R. Roy 2015. "Finance Commission of India's Assessment: A Political Economy Contention between Expectations and Outcomes." Applied Economics 48(2): 73-88.

Pattnaik, R. K. 1990. "Fiscal Marksmanship in India." Reserve Bank of India Occasional Papers 11(3).

Pina, Á. M., and N. M. Venes. 2011. "The political economy of EDP fiscal forecasts: An empirical assessment." European Journal of Political Economy 27(3): 534-46. 
Pindyck, R. S., and Rubinfeld, D. L. 1998 Econometric Models and Economic Forecasts. New York: Irwin/McGraw-Hill.

Rullán, X. O., and J. R. Villalonga. 2018. "Determinants of Regional Budget Forecast Errors in Federal Economies: Spain 1995-2013*.” Review of Public Economics 226(4): 85121.

Samuel, P., and C. Rangarajan. 1974. Short-Term Investment Forecasting. Noida: Macmillan India.

Shrestha, R., and L. Chakraborty. 2019. "Practising Subnational Public Finance in an Emerging Economy: Fiscal Marksmanship in Kerala.” NIPFP Working Paper No. 261. New Delhi: National Institute of Public Finance and Policy.

Strauch, R., M. Hallerberg, and J. v. Hagen. 2004. "Budgetary Forecasts in Europe - The Track Record of Stability and Convergence Programmes" ECB Working Paper No. 307. Germany: European Central Bank (ECB)

Theil, H. 1958. Economic Forecasts and Policy. Amsterdam: North Holland. 


\section{APPENDIX 1: STATEWISE PARTITIONING OF THE ERRORS}

Table A1: Revenue Deficit: Sources of Forecast Errors (BE-Actuals and RE-Actuals)

\begin{tabular}{|c|c|c|c|c|c|c|}
\hline & \multicolumn{3}{|c|}{ BE-Actuals } & \multicolumn{3}{|c|}{ RE-Actuals } \\
\hline & \multicolumn{3}{|c|}{ Sources of Error } & \multicolumn{3}{|c|}{ Sources of Error } \\
\hline & Bias & Variance & Random & Bias & Variance & Random \\
\hline Andhra Pradesh & 0.375 & 0.267 & 0.358 & 0.264 & 0.570 & 0.165 \\
\hline Arunachal Pradesh & 0.437 & 0.006 & 0.556 & 0.007 & 0.166 & 0.826 \\
\hline Assam & 0.193 & 0.013 & 0.794 & 0.461 & 0.324 & 0.215 \\
\hline Bihar & 0.394 & 0.089 & 0.517 & 0.437 & 0.051 & 0.512 \\
\hline Chhattisgarh & 0.247 & 0.250 & 0.503 & 0.035 & 0.226 & 0.739 \\
\hline Goa & 0.206 & 0.249 & 0.544 & 0.723 & 0.157 & 0.120 \\
\hline Gujarat & 0.001 & 0.105 & 0.895 & 0.013 & 0.085 & 0.902 \\
\hline Haryana & 0.587 & 0.206 & 0.207 & 0.000 & 0.491 & 0.509 \\
\hline Himachal & 0.294 & 0.001 & 0.705 & 0.163 & 0.170 & 0.668 \\
\hline Jammu and Kashmir & 0.448 & 0.384 & 0.167 & 0.333 & 0.348 & 0.319 \\
\hline Jharkhand & 0.707 & 0.001 & 0.292 & 0.366 & 0.114 & 0.520 \\
\hline Karnataka & 0.432 & 0.524 & 0.044 & 0.600 & 0.246 & 0.153 \\
\hline Kerala & 0.056 & 0.711 & 0.233 & 0.073 & 0.534 & 0.394 \\
\hline Madhya Pradesh & 0.396 & 0.115 & 0.489 & 0.444 & 0.123 & 0.433 \\
\hline Maharashtra & 0.385 & 0.393 & 0.222 & 0.025 & 0.129 & 0.845 \\
\hline Manipur & 0.000 & 0.018 & 0.982 & 0.018 & 0.308 & 0.787 \\
\hline Meghalaya & 0.767 & 0.022 & 0.210 & 0.668 & 0.000 & 0.332 \\
\hline Mizoram & 0.626 & 0.208 & 0.166 & 0.108 & 0.001 & 0.891 \\
\hline Nagaland & 0.498 & 0.327 & 0.175 & 0.343 & 0.235 & 0.423 \\
\hline Orissa & 0.892 & 0.023 & 0.085 & 0.897 & 0.001 & 0.102 \\
\hline Punjab & 0.710 & 0.185 & 0.105 & 0.080 & 0.371 & 0.548 \\
\hline Rajasthan & 0.270 & 0.516 & 0.214 & 0.500 & 0.315 & 0.185 \\
\hline Sikkim & 0.893 & 0.000 & 0.106 & 0.937 & 0.002 & 0.061 \\
\hline Tamil Nadu & 0.258 & 0.005 & 0.738 & 0.029 & 0.001 & 0.970 \\
\hline Tripura & 0.063 & 0.673 & 0.265 & 0.042 & 0.234 & 0.723 \\
\hline Uttarakhand & 0.176 & 0.260 & 0.564 & 0.154 & 0.651 & 0.195 \\
\hline Uttar Pradesh & 0.076 & 0.619 & 0.305 & 0.286 & 0.075 & 0.639 \\
\hline West Bengal & 0.873 & 0.001 & 0.127 & 0.389 & 0.005 & 0.606 \\
\hline
\end{tabular}

Source: CAG office finance accounts of states and state budget documents (basic data; various years) 
Table A2: Fiscal Deficit: Sources of Forecast Errors (BE-Actuals and RE-Actuals)

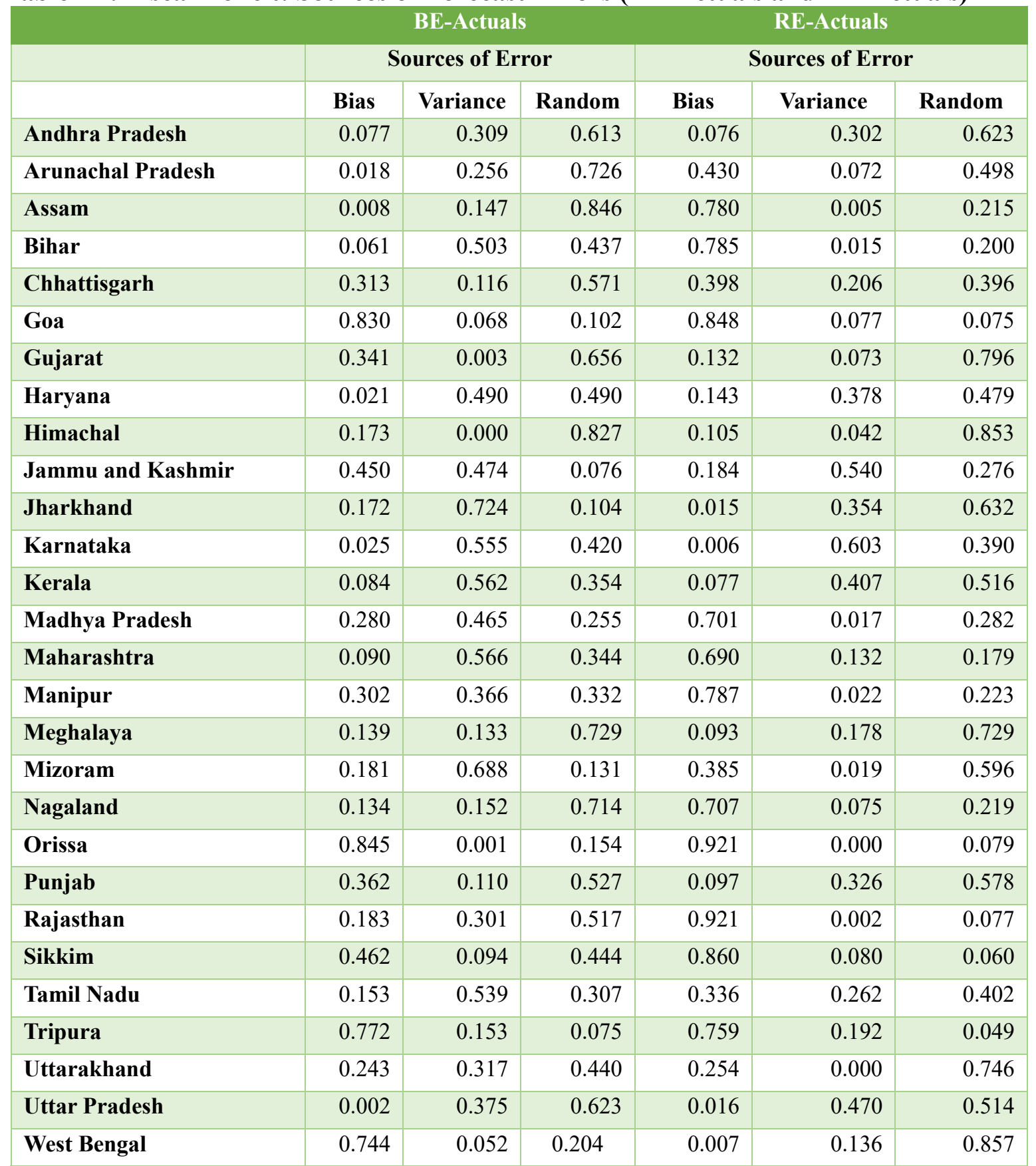

Source: CAG office finance accounts of states and state budget documents (basic data; various years) 
Table A3: Primary Deficit: Sources of Forecast Errors (BE-Actuals and RE-Actuals)

\begin{tabular}{|c|c|c|c|c|c|c|}
\hline & \multicolumn{3}{|c|}{ BE-Actuals } & \multicolumn{3}{|c|}{ RE-Actuals } \\
\hline & \multicolumn{3}{|c|}{ Sources of Error } & \multicolumn{3}{|c|}{ Sources of Error } \\
\hline & Bias & Variance & Random & Bias & Variance & Random \\
\hline Andhra Pradesh & 0.224 & 0.510 & 0.266 & 0.112 & 0.504 & 0.384 \\
\hline Arunachal Pradesh & 0.024 & 0.320 & 0.655 & 0.430 & 0.063 & 0.507 \\
\hline Assam & 0.009 & 0.137 & 0.854 & 0.777 & 0.003 & 0.219 \\
\hline Bihar & 0.123 & 0.524 & 0.352 & 0.778 & 0.024 & 0.199 \\
\hline Chhattisgarh & 0.303 & 0.218 & 0.479 & 0.373 & 0.311 & 0.316 \\
\hline Goa & 0.853 & 0.017 & 0.129 & 0.867 & 0.033 & 0.100 \\
\hline Gujarat & 0.309 & 0.087 & 0.604 & 0.116 & 0.225 & 0.659 \\
\hline Haryana & 0.032 & 0.497 & 0.471 & 0.142 & 0.353 & 0.504 \\
\hline Himachal & 0.124 & 0.025 & 0.851 & 0.047 & 0.087 & 0.866 \\
\hline Jammu and Kashmir & 0.381 & 0.552 & 0.067 & 0.180 & 0.594 & 0.227 \\
\hline Jharkhand & 0.166 & 0.644 & 0.189 & 0.016 & 0.261 & 0.723 \\
\hline Karnataka & 0.200 & 0.379 & 0.420 & 0.008 & 0.693 & 0.299 \\
\hline Kerala & 0.084 & 0.693 & 0.223 & 0.080 & 0.578 & 0.343 \\
\hline Madhya Pradesh & 0.118 & 0.584 & 0.299 & 0.666 & 0.001 & 0.333 \\
\hline Maharashtra & 0.072 & 0.545 & 0.383 & 0.692 & 0.090 & 0.218 \\
\hline Manipur & 0.337 & 0.341 & 0.322 & 0.787 & 0.015 & 0.231 \\
\hline Meghalaya & 0.142 & 0.304 & 0.554 & 0.099 & 0.357 & 0.544 \\
\hline Mizoram & 0.283 & 0.569 & 0.148 & 0.351 & 0.006 & 0.643 \\
\hline Nagaland & 0.028 & 0.019 & 0.954 & 0.714 & 0.007 & 0.280 \\
\hline Orissa & 0.632 & 0.005 & 0.363 & 0.637 & 0.015 & 0.348 \\
\hline Punjab & 0.360 & 0.078 & 0.562 & 0.098 & 0.331 & 0.571 \\
\hline Rajasthan & 0.183 & 0.363 & 0.454 & 0.915 & 0.000 & 0.085 \\
\hline Sikkim & 0.437 & 0.119 & 0.444 & 0.851 & 0.093 & 0.056 \\
\hline Tamil Nadu & 0.170 & 0.590 & 0.241 & 0.313 & 0.301 & 0.386 \\
\hline Tripura & 0.795 & 0.130 & 0.075 & 0.689 & 0.279 & 0.033 \\
\hline Uttarakhand & 0.231 & 0.389 & 0.380 & 0.288 & 0.000 & 0.711 \\
\hline Uttar Pradesh & 0.017 & 0.399 & 0.584 & 0.025 & 0.521 & 0.454 \\
\hline West Bengal & 0.761 & 0.001 & 0.239 & 0.002 & 0.172 & 0.826 \\
\hline
\end{tabular}

Source: CAG office finance accounts of states and state budget documents (basic data; various years) 
Table A4: Revenue Receipts: Sources of Forecast Errors (BE-Actuals and RE-Actuals)

\begin{tabular}{|c|c|c|c|c|c|c|}
\hline & & BE-Actual & & & RE-Actuals & \\
\hline & & urces of Ei & & & urces of Err & \\
\hline & Bias & Variance & Random & Bias & Variance & Random \\
\hline Andhra Pradesh & 0.478 & 0.191 & 0.331 & 0.365 & 0.375 & 0.260 \\
\hline Arunachal Pradesh & 0.482 & 0.004 & 0.513 & 0.286 & 0.078 & 0.635 \\
\hline Assam & 0.750 & 0.217 & 0.033 & 0.847 & 0.023 & 0.130 \\
\hline Bihar & 0.773 & 0.081 & 0.146 & 0.657 & 0.187 & 0.156 \\
\hline Chhattisgarh & 0.672 & 0.219 & 0.109 & 0.637 & 0.287 & 0.075 \\
\hline Goa & 0.772 & 0.068 & 0.160 & 0.721 & 0.051 & 0.229 \\
\hline Gujarat & 0.022 & 0.008 & 0.970 & 0.061 & 0.131 & 0.807 \\
\hline Haryana & 0.874 & 0.032 & 0.094 & 0.785 & 0.033 & 0.181 \\
\hline Himachal & 0.071 & 0.283 & 0.647 & 0.087 & 0.510 & 0.404 \\
\hline Jammu and Kashmir & 0.403 & 0.100 & 0.497 & 0.092 & 0.050 & 0.859 \\
\hline Jharkhand & 0.344 & 0.061 & 0.595 & 0.080 & 0.083 & 0.836 \\
\hline Karnataka & 0.073 & 0.151 & 0.776 & 0.144 & 0.273 & 0.582 \\
\hline Kerala & 0.380 & 0.001 & 0.619 & 0.004 & 0.290 & 0.706 \\
\hline Madhya Pradesh & 0.904 & 0.032 & 0.065 & 0.217 & 0.062 & 0.721 \\
\hline Maharashtra & 0.962 & 0.000 & 0.038 & 0.041 & 0.092 & 0.867 \\
\hline Manipur & 0.711 & 0.024 & 0.265 & 0.491 & 0.016 & 0.493 \\
\hline Meghalaya & 0.718 & 0.162 & 0.120 & 0.272 & 0.308 & 0.421 \\
\hline Mizoram & 0.007 & 0.015 & 0.977 & 0.027 & 0.501 & 0.472 \\
\hline Nagaland & 0.764 & 0.139 & 0.098 & 0.610 & 0.202 & 0.188 \\
\hline Orissa & 0.078 & 0.013 & 0.909 & 0.136 & 0.155 & 0.709 \\
\hline Punjab & 0.394 & 0.142 & 0.463 & 0.143 & 0.284 & 0.573 \\
\hline Rajasthan & 0.050 & 0.016 & 0.935 & 0.181 & 0.195 & 0.624 \\
\hline Sikkim & 0.006 & 0.650 & 0.343 & 0.017 & 0.427 & 0.556 \\
\hline Tamil Nadu & 0.895 & 0.020 & 0.085 & 0.078 & 0.038 & 0.884 \\
\hline Tripura & 0.363 & 0.599 & 0.038 & 0.016 & 0.022 & 0.961 \\
\hline Uttarakhand & 0.762 & 0.194 & 0.044 & 0.640 & 0.139 & 0.221 \\
\hline Uttar Pradesh & 0.651 & 0.124 & 0.225 & 0.072 & 0.003 & 0.925 \\
\hline West Bengal & 0.795 & 0.112 & 0.092 & 0.373 & 0.301 & 0.326 \\
\hline
\end{tabular}

Source: CAG office finance accounts of states and state budget documents (basic data; various years) 
Table A5: Own Tax Revenue: Sources of Forecast Errors (BE-Actuals and RE-Actuals)

\begin{tabular}{|c|c|c|c|c|c|c|}
\hline & & BE-Actual & & & RE-Actuals & \\
\hline & & urces of Er & & & urces of Err & \\
\hline & Bias & Variance & Random & Bias & Variance & Random \\
\hline Andhra Pradesh & 0.434 & 0.054 & 0.513 & 0.335 & 0.190 & 0.475 \\
\hline Arunachal Pradesh & 0.102 & 0.401 & 0.497 & 0.135 & 0.122 & 0.743 \\
\hline Assam & 0.182 & 0.781 & 0.037 & 0.205 & 0.476 & 0.319 \\
\hline Bihar & 0.513 & 0.411 & 0.076 & 0.424 & 0.182 & 0.394 \\
\hline Chhattisgarh & 0.364 & 0.588 & 0.049 & 0.422 & 0.531 & 0.046 \\
\hline Goa & 0.663 & 0.210 & 0.128 & 0.500 & 0.057 & 0.443 \\
\hline Gujarat & 0.120 & 0.796 & 0.084 & 0.004 & 0.402 & 0.593 \\
\hline Haryana & 0.558 & 0.374 & 0.068 & 0.625 & 0.315 & 0.060 \\
\hline Himachal & 0.001 & 0.000 & 0.998 & 0.021 & 0.001 & 0.978 \\
\hline Jammu and Kashmir & 0.212 & 0.028 & 0.760 & 0.205 & 0.040 & 0.755 \\
\hline Jharkhand & 0.664 & 0.295 & 0.041 & 0.574 & 0.379 & 0.047 \\
\hline Karnataka & 0.285 & 0.576 & 0.139 & 0.729 & 0.000 & 0.271 \\
\hline Kerala & 0.796 & 0.123 & 0.081 & 0.691 & 0.098 & 0.212 \\
\hline Madhya Pradesh & 0.006 & 0.891 & 0.103 & 0.000 & 0.371 & 0.628 \\
\hline Maharashtra & 0.000 & 0.719 & 0.280 & 0.067 & 0.389 & 0.544 \\
\hline Manipur & 0.164 & 0.702 & 0.135 & 0.184 & 0.228 & 0.685 \\
\hline Meghalaya & 0.014 & 0.458 & 0.529 & 0.053 & 0.408 & 0.538 \\
\hline Mizoram & 0.391 & 0.326 & 0.283 & 0.346 & 0.447 & 0.207 \\
\hline Nagaland & 0.272 & 0.434 & 0.294 & 0.365 & 0.016 & 0.619 \\
\hline Orissa & 0.068 & 0.001 & 0.931 & 0.163 & 0.000 & 0.837 \\
\hline Punjab & 0.864 & 0.048 & 0.088 & 0.873 & 0.092 & 0.034 \\
\hline Rajasthan & 0.084 & 0.859 & 0.058 & 0.162 & 0.795 & 0.044 \\
\hline Sikkim & 0.649 & 0.007 & 0.344 & 0.534 & 0.117 & 0.349 \\
\hline Tamil Nadu & 0.434 & 0.385 & 0.181 & 0.601 & 0.111 & 0.288 \\
\hline Tripura & 0.001 & 0.368 & 0.631 & 0.146 & 0.150 & 0.705 \\
\hline Uttarakhand & 0.589 & 0.383 & 0.028 & 0.421 & 0.444 & 0.135 \\
\hline Uttar Pradesh & 0.019 & 0.879 & 0.102 & 0.115 & 0.164 & 0.721 \\
\hline West Bengal & 0.693 & 0.063 & 0.244 & 0.438 & 0.106 & 0.456 \\
\hline
\end{tabular}

Source: CAG office finance accounts of states and state budget documents (basic data; various years) 
Table A6: Tax Transfers: Sources of Forecast Errors (BE-Actuals and RE-Actuals)

\begin{tabular}{|c|c|c|c|c|c|c|}
\hline & \multicolumn{3}{|c|}{ BE-Actuals } & \multicolumn{3}{|c|}{ RE-Actuals } \\
\hline & \multicolumn{3}{|c|}{ Sources of Error } & \multicolumn{3}{|c|}{ Sources of Error } \\
\hline & Bias & Variance & Random & Bias & Variance & Random \\
\hline Andhra Pradesh & 0.230 & 0.002 & 0.768 & 0.362 & 0.000 & 0.638 \\
\hline Arunachal Pradesh & 0.435 & 0.286 & 0.279 & 0.039 & 0.004 & 0.957 \\
\hline Assam & 0.001 & 0.251 & 0.748 & 0.055 & 0.117 & 0.828 \\
\hline Bihar & 0.387 & 0.004 & 0.609 & 0.391 & 0.017 & 0.591 \\
\hline Chhattisgarh & 0.391 & 0.036 & 0.572 & 0.420 & 0.000 & 0.580 \\
\hline Goa & 0.001 & 0.094 & 0.905 & 0.109 & 0.159 & 0.732 \\
\hline Gujarat & 0.006 & 0.124 & 0.871 & 0.653 & 0.022 & 0.326 \\
\hline Haryana & 0.142 & 0.288 & 0.571 & 0.491 & 0.216 & 0.293 \\
\hline Himachal & 0.458 & 0.001 & 0.541 & 0.402 & 0.009 & 0.588 \\
\hline Jammu and Kashmir & 0.223 & 0.000 & 0.777 & 0.171 & 0.002 & 0.828 \\
\hline Jharkhand & 0.047 & 0.220 & 0.732 & 0.119 & 0.279 & 0.602 \\
\hline Karnataka & 0.092 & 0.146 & 0.762 & 0.261 & 0.022 & 0.717 \\
\hline Kerala & 0.079 & 0.084 & 0.837 & 0.279 & 0.020 & 0.701 \\
\hline Madhya Pradesh & 0.075 & 0.215 & 0.710 & 0.321 & 0.009 & 0.670 \\
\hline Maharashtra & 0.175 & 0.257 & 0.568 & 0.037 & 0.090 & 0.873 \\
\hline Manipur & 0.707 & 0.004 & 0.288 & 0.332 & 0.115 & 0.645 \\
\hline Meghalaya & 0.727 & 0.004 & 0.269 & 0.434 & 0.071 & 0.495 \\
\hline Mizoram & 0.000 & 0.142 & 0.857 & 0.018 & 0.006 & 0.976 \\
\hline Nagaland & 0.018 & 0.243 & 0.739 & 0.143 & 0.073 & 0.784 \\
\hline Orissa & 0.112 & 0.182 & 0.706 & 0.048 & 0.028 & 0.924 \\
\hline Punjab & 0.090 & 0.461 & 0.449 & 0.213 & 0.018 & 0.768 \\
\hline Rajasthan & 0.215 & 0.173 & 0.612 & 0.143 & 0.010 & 0.847 \\
\hline Sikkim & 0.651 & 0.002 & 0.346 & 0.317 & 0.005 & 0.678 \\
\hline Tamil Nadu & 0.263 & 0.202 & 0.535 & 0.423 & 0.012 & 0.566 \\
\hline Tripura & 0.164 & 0.532 & 0.304 & 0.168 & 0.523 & 0.309 \\
\hline Uttarakhand & 0.023 & 0.224 & 0.753 & 0.057 & 0.052 & 0.891 \\
\hline Uttar Pradesh & 0.169 & 0.305 & 0.526 & 0.332 & 0.001 & 0.667 \\
\hline West Bengal & 0.006 & 0.469 & 0.525 & 0.214 & 0.003 & 0.783 \\
\hline
\end{tabular}

Source: CAG office finance accounts of states and state budget documents (basic data; various years) 
Table A7: Own Nontax Revenue: Sources of Forecast Errors (BE-Actuals and REActuals)

\begin{tabular}{|c|c|c|c|c|c|c|}
\hline & & BE-Actual & & & RE-Actuals & \\
\hline & & urces of Ei & & & urces of Err & \\
\hline & Bias & Variance & Random & Bias & Variance & Random \\
\hline Andhra Pradesh & 0.098 & 0.000 & 0.901 & 0.201 & 0.057 & 0.743 \\
\hline Arunachal Pradesh & 0.186 & 0.337 & 0.477 & 0.431 & 0.029 & 0.540 \\
\hline Assam & 0.620 & 0.157 & 0.223 & 0.375 & 0.000 & 0.625 \\
\hline Bihar & 0.796 & 0.013 & 0.191 & 0.268 & 0.051 & 0.681 \\
\hline Chhattisgarh & 0.652 & 0.268 & 0.080 & 0.546 & 0.351 & 0.103 \\
\hline Goa & 0.526 & 0.014 & 0.459 & 0.027 & 0.417 & 0.556 \\
\hline Gujarat & 0.047 & 0.060 & 0.893 & 0.240 & 0.335 & 0.425 \\
\hline Haryana & 0.446 & 0.363 & 0.191 & 0.503 & 0.179 & 0.318 \\
\hline Himachal & 0.007 & 0.070 & 0.923 & 0.018 & 0.042 & 0.940 \\
\hline Jammu and Kashmir & 0.207 & 0.017 & 0.776 & 0.320 & 0.055 & 0.625 \\
\hline Jharkhand & 0.429 & 0.316 & 0.255 & 0.453 & 0.375 & 0.172 \\
\hline Karnataka & 0.274 & 0.573 & 0.153 & 0.000 & 0.582 & 0.418 \\
\hline Kerala & 0.001 & 0.490 & 0.509 & 0.763 & 0.069 & 0.169 \\
\hline Madhya Pradesh & 0.009 & 0.166 & 0.824 & 0.189 & 0.016 & 0.795 \\
\hline Maharashtra & 0.522 & 0.340 & 0.138 & 0.719 & 0.072 & 0.209 \\
\hline Manipur & 0.923 & 0.063 & 0.014 & 0.536 & 0.118 & 0.404 \\
\hline Meghalaya & 0.188 & 0.000 & 0.811 & 0.121 & 0.013 & 0.866 \\
\hline Mizoram & 0.038 & 0.601 & 0.362 & 0.016 & 0.573 & 0.411 \\
\hline Nagaland & 0.726 & 0.163 & 0.110 & 0.431 & 0.016 & 0.552 \\
\hline Orissa & 0.204 & 0.549 & 0.247 & 0.388 & 0.314 & 0.298 \\
\hline Punjab & 0.127 & 0.073 & 0.801 & 0.651 & 0.019 & 0.331 \\
\hline Rajasthan & 0.010 & 0.414 & 0.576 & 0.067 & 0.281 & 0.651 \\
\hline Sikkim & 0.661 & 0.195 & 0.144 & 0.561 & 0.045 & 0.394 \\
\hline Tamil Nadu & 0.390 & 0.030 & 0.580 & 0.007 & 0.077 & 0.916 \\
\hline Tripura & 0.016 & 0.329 & 0.655 & 0.025 & 0.245 & 0.730 \\
\hline Uttarakhand & 0.261 & 0.435 & 0.304 & 0.224 & 0.237 & 0.540 \\
\hline Uttar Pradesh & 0.405 & 0.231 & 0.364 & 0.297 & 0.056 & 0.647 \\
\hline West Bengal & 0.344 & 0.056 & 0.600 & 0.150 & 0.050 & 0.800 \\
\hline
\end{tabular}

Source: CAG office finance accounts of states and state budget documents (basic data; various years) 
Table A8: Grants: Sources of Forecast Errors (BE-Actuals and RE-Actuals)

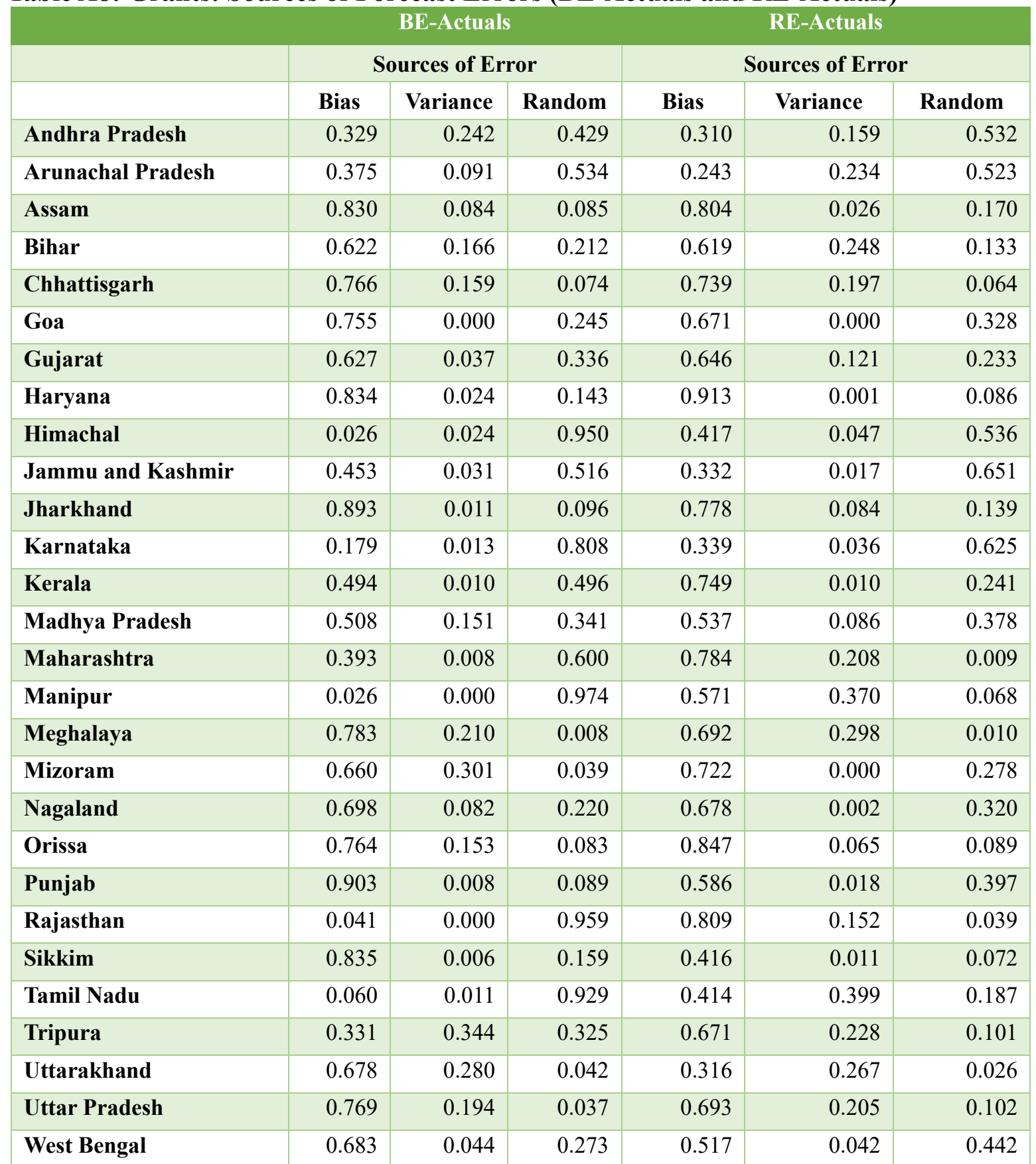

Source: CAG office finance accounts of states and state budget documents (basic data; various years) 
Table A9: Revenue Expenditure: Sources of Forecast Errors (BE-Actuals and REActuals)

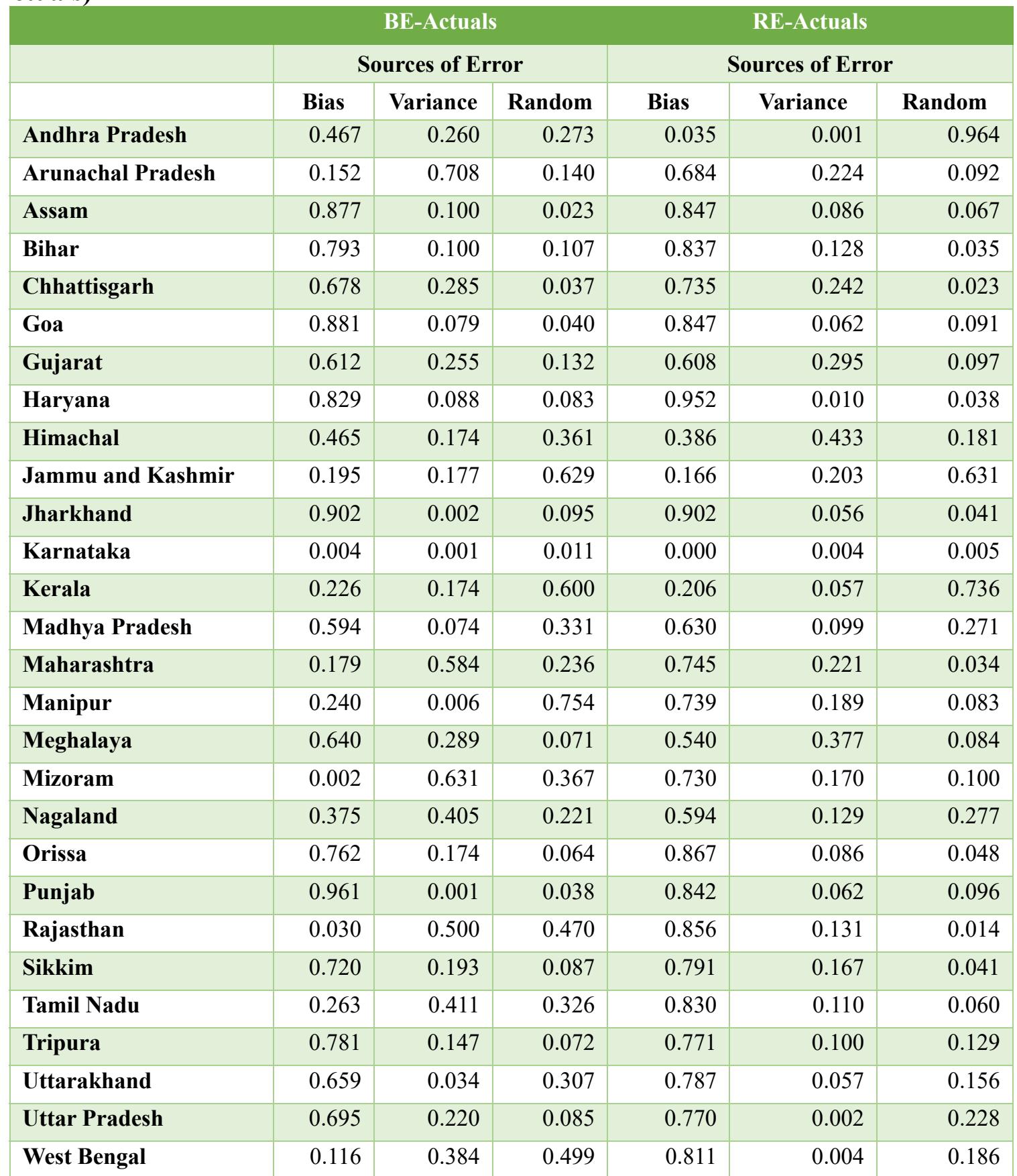

Source: CAG office finance accounts of states and state budget documents (basic data; various years) 
Table A10: Capital Expenditure: Sources of Forecast Errors (BE-Actuals and REActuals)

\begin{tabular}{|c|c|c|c|c|c|c|}
\hline & & BE-Actual & & & RE-Actuals & \\
\hline & & urces of E & & & urces of Err & \\
\hline & Bias & Variance & Random & Bias & Variance & Random \\
\hline Andhra Pradesh & 0.357 & 0.236 & 0.407 & 0.053 & 0.646 & 0.301 \\
\hline Arunachal Pradesh & 0.828 & 0.006 & 0.166 & 0.893 & 0.004 & 0.103 \\
\hline Assam & 0.813 & 0.112 & 0.075 & 0.767 & 0.167 & 0.066 \\
\hline Bihar & 0.756 & 0.003 & 0.241 & 0.738 & 0.009 & 0.254 \\
\hline Chhattisgarh & 0.894 & 0.073 & 0.033 & 0.851 & 0.122 & 0.027 \\
\hline Goa & 0.806 & 0.152 & 0.042 & 0.807 & 0.141 & 0.052 \\
\hline Gujarat & 0.481 & 0.199 & 0.321 & 0.638 & 0.172 & 0.190 \\
\hline Haryana & 0.105 & 0.008 & 0.887 & 0.018 & 0.046 & 0.936 \\
\hline Himachal & 0.196 & 0.005 & 0.800 & 0.554 & 0.317 & 0.129 \\
\hline Jammu and Kashmir & 0.443 & 0.035 & 0.522 & 0.461 & 0.041 & 0.498 \\
\hline Jharkhand & 0.728 & 0.205 & 0.067 & 0.791 & 0.070 & 0.139 \\
\hline Karnataka & 0.484 & 0.036 & 0.480 & 0.330 & 0.092 & 0.579 \\
\hline Kerala & 0.409 & 0.000 & 0.591 & 0.094 & 0.237 & 0.669 \\
\hline Madhya Pradesh & 0.317 & 0.532 & 0.152 & 0.315 & 0.085 & 0.600 \\
\hline Maharashtra & 0.980 & 0.001 & 0.019 & 0.696 & 0.252 & 0.052 \\
\hline Manipur & 0.463 & 0.256 & 0.281 & 0.906 & 0.050 & 0.051 \\
\hline Meghalaya & 0.854 & 0.127 & 0.019 & 0.777 & 0.192 & 0.031 \\
\hline Mizoram & 0.260 & 0.376 & 0.364 & 0.657 & 0.209 & 0.134 \\
\hline Nagaland & 0.918 & 0.004 & 0.079 & 0.722 & 0.154 & 0.123 \\
\hline Orissa & 0.024 & 0.910 & 0.065 & 0.064 & 0.179 & 0.757 \\
\hline Punjab & 0.797 & 0.001 & 0.202 & 0.909 & 0.007 & 0.084 \\
\hline Rajasthan & 0.167 & 0.061 & 0.772 & 0.872 & 0.013 & 0.115 \\
\hline Sikkim & 0.959 & 0.023 & 0.018 & 0.969 & 0.017 & 0.014 \\
\hline Tamil Nadu & 0.525 & 0.135 & 0.340 & 0.735 & 0.046 & 0.219 \\
\hline Tripura & 0.595 & 0.374 & 0.032 & 0.910 & 0.069 & 0.022 \\
\hline Uttarakhand & 0.027 & 0.047 & 0.926 & 0.529 & 0.094 & 0.378 \\
\hline Uttar Pradesh & 0.314 & 0.005 & 0.681 & 0.482 & 0.033 & 0.485 \\
\hline West Bengal & 0.864 & 0.041 & 0.095 & 0.854 & 0.140 & 0.006 \\
\hline
\end{tabular}

Source: CAG office finance accounts of states and state budget documents (basic data; various years) 